\title{
Donsker-Varadhan Large Deviations for Path-Distribution Dependent SPDEs *
}

\author{
Panpan Ren ${ }^{b, c)}$, Feng-Yu Wang ${ }^{a, b)}$ \\ a) Center for Applied Mathematics, Tianjin University, Tianjin 300072, China \\ b) Department of Mathematics, Swansea University, Bay Campus, SA1 8EN, United Kingdom \\ c) Mathematical Institute,Woodstock Road, OX2 6GG, University of Oxford \\ 673788@swansea.ac.uk, Panpan.ren@maths.ox.ac.uk; wangfy@tju.edu.cn, F.-Y.Wang@swansea.ac.uk
}

February 21, 2020

\begin{abstract}
As an important tool characterizing the long time behavior of Markov processes, the Donsker-Varadhan LDP (large deviation principle) does not directly apply to distribution dependent SDEs/SPDEs since the solutions are non-Markovian. We establish this type LDP for several different models of distribution dependent SDEs/SPDEs which may also with memories, by comparing the original equations with the corresponding distribution independent ones. As preparations, the existence, uniqueness and exponential convergence are also investigated for path-distribution dependent SPDEs which should be interesting by themselves.
\end{abstract}

AMS subject Classification: 60B05, 60B10.

Keywords: Donsker-Varadhan LDP, path-distribution dependent SDEs, Warsserstein distance.

\section{Introduction}

The LDP (large deviation principle) is a fundamental tool characterizing the asymptotic behaviour of probability measures $\left\{\mu_{\varepsilon}\right\}_{\varepsilon>0}$ on a topological space $E$, see [4] and references within. Recall that $\mu_{\varepsilon}$ for small $\varepsilon>0$ is said to satisfy the LDP with speed $\lambda(\varepsilon) \rightarrow+\infty$ (as $\left.\varepsilon \rightarrow 0\right)$ and rate function $I: E \rightarrow[0,+\infty]$, if $I$ has compact level sets (i.e. $\{I \leq r\}$ is compact for $r \in \mathbb{R}^{+}$), and for any Borel subset $A$ of $E$,

$$
-\inf _{A^{o}} I \leq \liminf _{\varepsilon \rightarrow 0} \frac{1}{\lambda(\varepsilon)} \log \mu_{\varepsilon}(A) \leq \limsup _{\varepsilon \rightarrow 0} \frac{1}{\lambda(\varepsilon)} \log \mu_{\varepsilon}(A) \leq-\inf _{\bar{A}} I,
$$

\footnotetext{
${ }^{*}$ Supported in part by NNSFC $(11771326,11831014)$.
} 
where $A^{o}$ and $\bar{A}$ stand for the interior and the closure of $A$ in $E$ respectively. The following two different type LDPs have been studied in the literature.

The Freidlin-Wentzell type small noise LDP [6]: $\mu_{\varepsilon}$ stands for the distribution of the solution to a dynamic system perturbed by a noise with small intensity $\varepsilon>0$, i.e. SDE (stochastic differential equation) with small noise. In this case, $E$ is the path space for the solutions of the SDE. This type LDP describes, as $\varepsilon \rightarrow 0$, the convergence of stochastic systems to the corresponding deterministic system.

The Donsker-Varadhan type long time LDP [5]: $\quad \mu_{\varepsilon}$ stands for the distribution of $L_{\varepsilon^{-1}}$, where

$$
L_{t}:=\frac{1}{t} \int_{0}^{t} \delta_{X(s)} \mathrm{d} s, \quad t>0
$$

is the empirical measure for a stochastic process $\{X(t)\}_{t \geq 0}$. This type LDP describes the behaviour of $L_{t}$ as $t \rightarrow \infty$. In this case, $E$ is the set of all probability measures on the state space of the process, on which both the weak topology (induced by bounded continuous functions) and the $\tau$-topology (induced by bounded measurable functions) are considered in the literature.

In this paper, we study the Donsker-Varadhan LDP for path-distribution dependent SDEs (stochastic differential equations) on a separable Hilbert space $\mathbb{H}$. Inspired by Kac's programme for Vlasov systems in kinetic theory [10], McKean [12] introduced distribution dependent SDEs. According to Sznitman [15], under the global Lipschtiz condition, these type SDEs can be derived as the limit of mean-field particle systems when the number of particles tends to infinity. Therefore, distribution dependent SDEs are also called Mckean-Vlasov SDEs and mean-field SDEs.

In applications, the distribution of a stochastic process can be regarded as a macro property, while the path of the process up to a time $t$ stands for the history of the system before this time. Since the evolution of a stochastic system may depend on both the macro environment and the history, it is reasonable to investigate path-distribution dependent SDEs. Moreover, because in many cases the configuration space for particle systems is infinite-dimensional, we consider path-distribution dependent SDEs on Hilbert spaces, and in this case the SDEs are called SPDEs (stochastic partial differential equations).

In recent years, distribution dependent SDEs have been intensively investigated. Among many other papers in this field, [13] established the Freidlin-Wentzell LDP for distribution dependent SDEs. However, up to our best knowledge, there is no any result on the DonskerVaradhan LDP for this type SDEs. Since the solution is non-Markovian, existing results on the Donsker-Varadhan LDP derived for Markov processes do not apply. Indeed, the definition of the rate function (the Donsker-Varadhan level 2 entropy function) depends on the Markov property of the process, for which the law of the process starting at an initial distribution $\nu$ is given by

$$
P^{\nu}=\int_{E} P^{x} \nu(\mathrm{d} x)
$$

where $P^{x}$ is the law of the process starting at $x$, see Subsection 3.2 for details. 
Main idea of the study. To establish the Donsker-Varadhan type LDP for a distribution dependent SDE/SPDE, we choose a reference SDE/SPDE whose solution is Markovian so that existing results on the Donsker-Varadhan LDP apply. By comparing the original equation with the reference one in the sense of LDP, see Lemma 3.5 below, we establish the Donsker-Varadhan LDP for the distribution dependent SDE/SPDE. To this end, we will assume that the original equation has a unique invariant probability measure $\bar{\mu}$, and take the reference equation to be the original one with $\bar{\mu}$ replacing the distribution variable.

The framework. For a measurable space $(E, \mathscr{B})$, let $\mathscr{P}(E)$ denote the set of all probability measures on $E$. For an $E$-valued random variable $\xi$ on a probability space, let $\mathscr{L}_{\xi} \in \mathscr{P}(E)$ be the distribution of $\xi$.

For a separable Hilbert space $\mathbb{H}$, let $\mathbb{L}(\mathbb{H})$ be the class of all bounded linear operators on $\mathbb{H}$, which is equipped with the operator norm $\|\cdot\|$. We will also use the Hilbert-Schmidt norm $\|\cdot\|_{H S}$. In general, for two separable Hilbert spaces $\mathbb{H}_{1}$ and $\mathbb{H}_{2}, \mathbb{L}_{(}\left(\mathbb{H}_{1} ; \mathbb{H}_{2}\right)$ stands for the space of all bounded linear operators from $\mathbb{H}_{1}$ to $\mathbb{H}_{2}$.

For a fixed constant $r_{0} \geq 0$, let $\mathscr{C}=C\left(\left[-r_{0}, 0\right] ; \mathbb{H}\right)$ be the space of all continuous maps from $\left[-r_{0}, 0\right]$ to $\mathbb{H}$ equipped with the uniform norm

$$
\|\xi\|_{\infty}:=\sup _{-r_{0} \leq \theta \leq 0}|\xi(\theta)|, \quad \xi \in \mathscr{C} .
$$

Then $\mathscr{C}$ is a Polish space, which refers to the history of a stochastic differential system on $\mathbb{H}$ with memory length $r_{0}$. When $r_{0}=0$, the path space $\mathscr{C}$ degenerates to $\mathbb{H}$. For any map $h(\cdot) \in C\left(\left[-r_{0}, \infty\right) ; \mathbb{H}\right)$ and $t \geq 0$, the corresponding segment $h_{t} \in \mathscr{C}$ is defined by

$$
h_{t}(r)=h(t+r), \quad r \in\left[-r_{0}, 0\right] \text {. }
$$

Let $W(t)$ be the cylindrical Brownian motion on $\tilde{\mathbb{H}}$ under a complete filtration probability space $\left(\Omega, \mathscr{F},\left\{\mathscr{F}_{t}\right\}_{t \geq 0}, \mathbb{P}\right)$; that is,

$$
W(t)=\sum_{i=1}^{\infty} B_{i}(t) \tilde{e}_{i}, \quad t \geq 0
$$

for an orthonormal basis $\left\{\tilde{e}_{i}\right\}_{i \geq 1}$ on $\tilde{\mathbb{H}}$ and a sequence of independent one-dimensional Brownian motions $\left\{B_{i}(t): t \geq 0\right\}_{i \geq 1}$ on $\left(\Omega, \mathscr{F},\left\{\mathscr{F}_{t}\right\}_{t \geq 0}, \mathbb{P}\right)$, where $\mathscr{F}_{0}$ is rich enough such that for any $\pi \in \mathscr{P}(\mathscr{C} \times \mathscr{C})$ there exists a $\mathscr{C} \times \mathscr{C}$-valued random variable $\xi$ on $\left(\Omega, \mathscr{F}_{0}, \mathbb{P}\right)$ such that $\mathscr{L}_{\xi}=\pi$. Consequently, for any $p>0$ and two probability measures

$$
\mu_{1}, \mu_{2} \in \mathscr{P}_{p}(\mathscr{C}):=\left\{\mu \in \mathscr{P}(\mathscr{C}):\|\mu\|_{p}:=\mu\left(\|\cdot\|_{\infty}^{p}\right)^{\frac{1}{p \vee 1}}<\infty\right\},
$$

there exist two $\mathscr{F}_{0}$-measurable random variables $\xi_{1}, \xi_{2}$ on $\mathscr{C}$ such that

$$
\left(\mathbb{E}\left[\left\|\xi_{1}-\xi_{2}\right\|_{\infty}^{p}\right]\right)^{\frac{1}{p}}=\mathbb{W}_{p}\left(\mu_{1}, \mu_{2}\right):=\inf _{\pi \in \mathscr{C}\left(\mu_{1}, \mu_{2}\right)}\left(\int_{\mathscr{C} \times \mathscr{C}}\|\xi-\eta\|_{\infty}^{p} \pi(\mathrm{d} \xi, \mathrm{d} \eta)\right)^{\frac{1}{p}}
$$

Note that $\mathscr{P}_{p}(\mathscr{C})$ is a Polish space under the $L^{p}$-Wasserstein distance $\left\{\mathbb{W}_{p}\right\}^{p \wedge 1}$. 
Now, consider the following path-distribution dependent SPDE on $\mathbb{H}$ :

*1 $\quad(1.1)$

$$
\mathrm{d} X(t)=\left\{A X(t)+b\left(X_{t}, \mathscr{L}_{X_{t}}\right)\right\} \mathrm{d} t+\sigma\left(\mathscr{L}_{X_{t}}\right) \mathrm{d} W(t), \quad t \geq 0,
$$

where $(A, \mathscr{D}(A))$ is a negative definite self-adjoint operator on $\mathbb{H}$,

$$
b: \mathscr{C} \times \mathscr{P}(\mathscr{C}) \rightarrow \mathbb{H}, \quad \sigma: \mathscr{P}(\mathscr{C}) \rightarrow \mathbb{L}(\tilde{\mathbb{H}} ; \mathbb{H})
$$

are measurable.

In Section 3, a more general equation (3.1) will be solved for initial value $X_{0} \in L^{p}(\Omega \rightarrow$ $\left.\mathscr{C}, \mathscr{F}_{0}, \mathbb{P}\right)$ for some $p>0$; i.e. $\mathscr{L}_{X_{0}}$ is in the space

However, to establish the Donsker-Varadhan LDP using the comparing method proposed in Theorem 4.1] below, we have to assume that the noise term only depends on the distribution $\mathscr{L}_{X_{t}}$ rather than the solution $X_{t}$.

Let $X_{t}^{\nu}$ denote the mild segment solution with initial distribution $\nu \in \mathscr{P}(\mathscr{C})$, which is a continuous adapted process on $\mathscr{C}$, see Definition 3.1 below for details. We study the long time LDP for the empirical measure

$$
L_{t}^{\nu}:=\frac{1}{t} \int_{0}^{t} \delta_{X_{s}^{\nu}} \mathrm{d} s, \quad t>0 .
$$

D2.1 Definition 1.1. Let $\mathscr{P}(\mathscr{C})$ be equipped with the weak topology, let $\mathscr{A} \subset \mathscr{P}(\mathscr{C})$, and let $J: \mathscr{P}(\mathscr{C}) \rightarrow[0, \infty]$ have compact level sets, i.e. $\{J \leq r\}$ is compact in $\mathscr{P}(\mathscr{C})$ for any $r>0$.

(1) $\left\{L_{t}^{\nu}\right\}_{\nu \in \mathscr{A}}$ is said to satisfy the upper bound uniform LDP with rate function $J$, denoted by $\left\{L_{t}^{\nu}\right\}_{\nu \in \mathscr{A}} \in L D P_{u}(J)$, if for any closed $A \subset \mathscr{P}(\mathscr{C})$,

$$
\limsup _{t \rightarrow \infty} \frac{1}{t} \sup _{\nu \in \mathscr{A}} \log \mathbb{P}\left(L_{t}^{\nu} \in A\right) \leq-\inf _{A} J .
$$

(2) $\left\{L_{t}^{\nu}\right\}_{\nu \in \mathscr{A}}$ is said to satisfy the lower bound uniform LDP with rate function $J$, denoted by $\left\{L_{t}^{\nu}\right\}_{\nu \in \mathscr{A}} \in L D P_{l}(J)$, if for any open $A \subset \mathscr{P}(\mathscr{C})$,

$$
\liminf _{t \rightarrow \infty} \frac{1}{t} \inf _{\nu \in \mathscr{A}} \log \mathbb{P}\left(L_{t}^{\nu} \in A\right) \geq-\inf _{A} J
$$

(3) $\left\{L_{t}^{\nu}\right\}_{\nu \in \mathscr{A}}$ is said to satisfy the uniform LDP with rate function $J$, denoted by $\left\{L_{t}^{\nu}\right\}_{\nu \in \mathscr{A}} \in$ $L D P(J)$, if $\left\{L_{t}^{\nu}\right\}_{\nu \in \mathscr{A}} \in L D P_{u}(J)$ and $\left\{L_{t}^{\nu}\right\}_{\nu \in \mathscr{A}} \in L D P_{l}(J)$.

The remainder of the paper is orgnized as follows. In Section 2, we state the main results of the paper and illustrate them by specific examples. To prove these results, in Section 3 we investigate the existence and uniqueness for path-distribution dependent SDEs/SPDEs, and recall some results on the Donsker-Varadhan LDP for Markov processes. Finally, the proofs of main results are addressed in Section 4. 


\section{Main results and Examples}

We investigate the long time LDP for (1.1) in the following three situations respectively:

1) $r_{0}=0$ and $\mathbb{H}$ is finite-dimensional;

2) $r_{0}=0$ and $\mathbb{H}$ is infinite-dimensional;

3) $r_{0}>0$ and $\sigma$ is constant.

When $r_{0}>0$ and $\sigma$ is non-constant, there is a difficulty to apply our comparison argument. We leave this for the future study.

To state our main results, we recall the Feller property, the strong Feller property and the irreducibility for a (sub-) Markov operator $P$. Let $\mathscr{B}_{b}(\mathscr{C})\left(\right.$ resp. $C_{b}(\mathscr{C})$ ) be the space of bounded measurable (resp. continuous) real functions on $\mathscr{C}$. Let $P$ be a sub-Markov operator on $\mathscr{B}_{b}(\mathscr{C})$, i.e. it is a positivity-preserving linear operator with $P 1 \leq 1$. $P$ is called strong Feller if $P \mathscr{B}_{b}(\mathscr{C}) \subset C_{b}(\mathscr{C})$, is called Feller if $P C_{b}(\mathscr{C}) \subset C_{b}(\mathscr{C})$, and is called $\mu$-irreducible for some $\mu \in \mathscr{P}(\mathscr{C})$ if $\mu\left(1_{A} P 1_{B}\right)>0$ holds for any $A, B \in \mathscr{B}(\mathscr{C})$ with $\mu(A) \mu(B)>0$.

\subsection{Distribution dependent SDE on $\mathbb{R}^{d}$}

Let $r_{0}=0, \mathbb{H}=\mathbb{R}^{d}$ and $\tilde{\mathbb{H}}=\mathbb{R}^{m}$ for some $d, m \in \mathbb{N}$. In this case, we combine the linear term $A x$ with the drift term $b(x, \mu)$, so that (1.1) reduces to

$$
\mathrm{d} X(t)=b\left(X(t), \mathscr{L}_{X(t)}\right) \mathrm{d} t+\sigma\left(\mathscr{L}_{X(t)}\right) \mathrm{d} W(t),
$$

where $b: \mathbb{R}^{d} \times \mathscr{P}_{2}\left(\mathbb{R}^{d}\right) \rightarrow \mathbb{R}^{d}, \sigma: \mathscr{P}_{2}\left(\mathbb{R}^{d}\right) \rightarrow \mathbb{R}^{d} \otimes \mathbb{R}^{m}$ and $W(t)$ is the $m$-dimensional Brownian motion. We assume

$\left(H_{1}\right) b$ is continuous, $\sigma$ is bounded and continuous such that

$$
2\langle b(x, \mu)-b(y, \nu), x-y\rangle+\|\sigma(\mu)-\sigma(\nu)\|_{H S}^{2} \leq-\kappa_{1}|x-y|^{2}+\kappa_{2} \mathbb{W}_{2}(\mu, \nu)^{2}
$$

holds for some constants $\kappa_{1}>\kappa_{2} \geq 0$ and all $x, y \in \mathbb{R}^{d}, \mu, \nu \in \mathscr{P}_{2}\left(\mathbb{R}^{d}\right)$.

Under $\left(H_{1}\right)$, for any $X(0) \in L^{2}\left(\Omega \rightarrow \mathbb{R}^{d}, \mathscr{F}_{0}, \mathbb{P}\right)$, the equation (2.1) has a unique solution, see [18, Theorem 2.1] or Theorem 3.1] in a more general framework. We write $P_{t}^{*} \mu=\mathscr{L}_{X(t)}$ if $\mathscr{L}_{X(0)}=\mu$. By [18, Theorem 3.1(2)], $P_{t}^{*}$ has a unique invariant probability measure $\bar{\mu} \in \mathscr{P}_{2}\left(\mathbb{R}^{d}\right)$ such that

$$
\mathbb{W}_{2}\left(P_{t}^{*} \nu, \bar{\mu}\right)^{2} \leq \mathrm{e}^{-\left(\kappa_{1}-\kappa_{2}\right) t} \mathbb{W}_{2}(\nu, \bar{\mu})^{2}, \quad t \geq 0, \nu \in \mathscr{P}_{2}\left(\mathbb{R}^{d}\right)
$$

Consider the reference SDE

$$
\mathrm{d} \bar{X}(t)=b(\bar{X}(t), \bar{\mu}) \mathrm{d} t+\sigma(\bar{\mu}) \mathrm{d} W(t) .
$$

It is standard that under $\left(H_{1}\right)$ the equation (2.3) has a unique solution $\bar{X}^{x}(t)$ for any starting point $x \in \mathbb{R}^{d}$, and $\bar{\mu}$ is the unique invariant probability measure of the associated Markov semigroup

$$
\bar{P}_{t} f(x):=\mathbb{E}\left[f\left(\bar{X}^{x}(t)\right)\right], \quad t \geq 0, x \in \mathbb{R}^{d}, f \in \mathscr{B}_{b}\left(\mathbb{R}^{d}\right)
$$


Consequently, $\bar{P}_{t}$ uniquely extends to $L^{\infty}(\bar{\mu})$. If $f \in L^{\infty}(\bar{\mu})$ satisfies

$$
\bar{P}_{t} f=f+\int_{0}^{t} \bar{P}_{s} g \mathrm{~d} s, \quad \bar{\mu} \text {-a.e. }
$$

for some $g \in L^{\infty}(\bar{\mu})$ and all $t \geq 0$, we write $f \in \mathscr{D}(\overline{\mathscr{A}})$ and denote $\overline{\mathscr{A}} f=g$. Obviously, we have $\mathscr{D}(\overline{\mathscr{A}}) \supset C_{c}^{\infty}\left(\mathbb{R}^{d}\right):=\left\{f \in C_{b}^{\infty}\left(\mathbb{R}^{d}\right): \nabla f\right.$ has compact support $\}$ and

$$
\overline{\mathscr{A}} f(x)=\frac{1}{2} \sum_{i, j=1}^{d}\left\{\sigma \sigma^{*}\right\}_{i j}(\bar{\mu}) \partial_{i} \partial_{j} f(x)+\sum_{i=1}^{\infty} b_{i}(x, \bar{\mu}) \partial_{i} f(x), \quad f \in C_{c}^{\infty}\left(\mathbb{R}^{d}\right) .
$$

According to Section 3, the Donsker-Varadhan level 2 entropy function $J$ for the diffusion process generated by $\overline{\mathscr{A}}$ has compact level sets in $\mathscr{P}\left(\mathbb{R}^{d}\right)$ under the $\tau$ and weak topologies, and by (3.11) below we have

$$
J(\nu)= \begin{cases}\sup \left\{\int_{\mathbb{R}^{d}} \frac{-\overline{\mathscr{A}} f}{f} \mathrm{~d} \nu: 1 \leq f \in \mathscr{D}(\overline{\mathscr{A}})\right\}, & \text { if } \nu \ll \mu \\ \infty, & \text { otherwise }\end{cases}
$$

T01 Theorem 2.1. Assume $\left(H_{1}\right)$. For any $r, R>0$, let $\mathscr{B}_{r, R}=\left\{\nu \in \mathscr{P}\left(\mathbb{R}^{d}\right): \nu\left(\mathrm{e}^{|\cdot|^{r}}\right) \leq R\right\}$.

(1) We have $\left\{L_{t}^{\nu}\right\}_{\nu \in \mathscr{B}_{r, R}} \in L D P_{u}(J)$ for all $r, R>0$. If $\bar{P}_{t}$ is strong Feller and $\bar{\mu}$-irreducible for some $t>0$, then $\left\{L_{t}^{\nu}\right\}_{\nu \in \mathscr{B}_{r, R}} \in L D P(J)$ for all $r, R>0$.

(2) If there exist constants $\varepsilon, c_{1}, c_{2}>0$ such that

$$
\langle x, b(x, \nu)\rangle \leq c_{1}-c_{2}|x|^{2+\varepsilon}, \quad x \in \mathbb{R}^{d}, \nu \in \mathscr{P}_{2}\left(\mathbb{R}^{d}\right)
$$

then $\left\{L_{t}^{\nu}\right\}_{\nu \in \mathscr{P}_{2}\left(\mathbb{R}^{d}\right)} \in L D P_{u}(J)$. If moreover $\bar{P}_{t}$ is strong Feller and $\bar{\mu}$-irreducible for some $t>0$, then $\left\{L_{t}^{\nu}\right\}_{\nu \in \mathscr{P}_{2}\left(\mathbb{R}^{d}\right)} \in L D P(J)$.

To apply this result, we first recall some facts on the strong Feller property and the irreducibility of diffusion semigroups.

Remark 2.1. (1) Let $\bar{P}_{t}$ be the (sub-)Markov semigroup generated by the second order differential operator

$$
\overline{\mathscr{A}}:=\sum_{i=1}^{m} U_{i}^{2}+U_{0}
$$

where $\left\{U_{i}\right\}_{i=1}^{m}$ are $C^{1}$-vector fields and $U_{0}$ is a continuous vector field. According to [11, Theorem 5.1], if $\left\{U_{i}: 1 \leq i \leq m\right\}$ together with their Lie brackets with $U_{0}$ span $\mathbb{R}^{d}$ at any point (i.e. the Hörmander condition holds), then the Harnack inequality

$$
P_{t} f(x) \leq \psi(t, s, x, y) P_{t+s} f(y), \quad t, s>0, x, y \in \mathbb{R}^{d}, f \in \mathscr{B}^{+}\left(\mathbb{R}^{d}\right)
$$

for some map $\psi:(0, \infty)^{2} \times\left(\mathbb{R}^{d}\right)^{2} \rightarrow(0, \infty)$. Consequently, if moreover $\bar{P}_{t}$ has an invariant probability measure $\bar{\mu}$, then $\bar{P}_{t}$ is $\bar{\mu}$-irreducible for any $t>0$. Finally, if $\left\{U_{i}\right\}_{0 \leq i \leq m}$ are smooth 
with bounded derivatives of all orders, then the above Hörmander condition implies that $\bar{P}_{t}$ has smooth heat kernel with respect to the Lebesgue measure, in particular it is strong Feller for any $t>0$.

(2) Let $\bar{P}_{t}$ be the Markov semigroup generated by

$$
\overline{\mathscr{A}}:=\sum_{i, j=1}^{d} \bar{a}_{i j} \partial_{i} \partial_{j}+\sum_{i=1}^{d} \bar{b}_{i} \partial_{j}
$$

where $\left(\bar{a}_{i j}(x)\right)$ is strictly positive definite for any $x, \bar{a}_{i j} \in H_{l o c}^{p, 1}(\mathrm{~d} x)$ and $\bar{b}_{i} \in L_{l o c}^{p}(\mathrm{~d} x)$ for some $p>d$ and all $1 \leq i, j \leq d$. Moreover, let $\bar{\mu}$ be an invariant probability measure of $\bar{P}_{t}$. Then by [2, Theorem 4.1], $\bar{P}_{t}$ is strong Feller for all $t>0$. Moreover, as indicated in (1) that [11, Theorem 5.1] ensures the $\bar{\mu}$-irreducibility of $\bar{P}_{t}$ for $t>0$.

We present below two examples to illustrate this result, where the first is a distribution dependent perturbation of the Ornstein-Ulenbeck process, and the second is the distribution dependent stochastic Hamiltonian system.

Example 2.1. Let $\sigma(\nu)=I+\varepsilon \sigma_{0}(\nu)$ and $b(x, \nu)=-\frac{1}{2}\left(\sigma \sigma^{*}\right)(\nu) x$, where $I$ is the identity matrix, $\varepsilon>0$ and $\sigma_{0}$ is a bounded Lipschitz continuous map from $\mathscr{P}_{2}\left(\mathbb{R}^{d}\right)$ to $\mathbb{R}^{d} \otimes \mathbb{R}^{d}$. When $\varepsilon>0$ is small enough, assumption $\left(H_{1}\right)$ holds and that $\bar{P}_{t}$ satisfies conditions in Remark 2.1(2). So, Theorem 2.1(1) implies $\left\{L_{t}^{\nu}\right\}_{\nu \in \mathscr{B}_{r, R}} \in L D P(J)$ for all $r, R>0$, where it is easy to see that the unique invariant probability measure $\bar{\mu}$ is the standard Gaussian measure on $\mathbb{R}^{d}$.

If we take $b(x, \nu)=-x-c|x|^{\theta} x$ for some constants $c, \theta>0$, then when $\varepsilon>0$ is small enough $\left(H_{1}\right)$ and (2.4) are satisfied, so that Theorem 2.1(2) and Remark 2.1(2) imply $\left\{L_{t}^{\nu}\right\}_{\nu \in \mathscr{P}_{2}\left(\mathbb{R}^{d}\right)} \in$ $L D P(J)$.

Example 2.2. Let $d=2 m$ and consider the following distribution dependent SDE for $X(t)=$ $\left(X^{(1)}(t), X^{(2)}(t)\right)$ on $\mathbb{R}^{m} \times \mathbb{R}^{m}$ :

$$
\left\{\begin{array}{l}
\mathrm{d} X^{(1)}(t)=\left\{X^{(2)}(t)-\lambda X^{(1)}(t)\right\} \mathrm{d} t \\
\mathrm{~d} X^{(2)}(t)=\left\{Z\left(X(t), \mathscr{L}_{X(t)}\right)-\lambda X^{(2)}(t)\right\} \mathrm{d} t+\sigma \mathrm{d} W(t),
\end{array}\right.
$$

were $\lambda>0$ is a constant, $\sigma$ is an invertible $m \times m$-matrix, $W(t)$ is the $m$-dimensional Brownian motion, and $Z: \mathbb{R}^{2 m} \times \mathscr{P}_{2}\left(\mathbb{R}^{2 m}\right) \rightarrow \mathbb{R}^{m}$ satisfies

$$
\left|Z\left(x_{1}, \nu_{1}\right)-Z\left(x_{2}, \nu_{2}\right)\right| \leq \alpha_{1}\left|x_{1}^{(1)}-x_{2}^{(1)}\right|+\alpha_{2}\left|x_{1}^{(2)}-x_{2}^{(2)}\right|+\alpha_{3} \mathbb{W}_{2}\left(\nu_{1}, \nu_{2}\right)
$$

for some constants $\alpha_{1}, \alpha_{2}, \alpha_{3} \geq 0$ and all $x_{i}=\left(x_{i}^{(1)}, x_{i}^{(2)}\right) \in \mathbb{R}^{2 m}, \nu_{i} \in \mathscr{P}_{2}\left(\mathbb{R}^{2 m}\right), 1 \leq i, j \leq 2$. If

$$
4 \lambda>\inf _{s>0}\left\{2 \alpha_{3} s+\alpha_{3} s^{-1}+2 \alpha_{2}+\sqrt{4\left(1+\alpha_{1}\right)^{2}+\left(2 \alpha_{2}+\alpha_{3} s^{-1}\right)^{2}}\right\},
$$

then $\left\{L_{t}^{\nu}\right\}_{\nu \in \mathscr{B}_{r, R}} \in L D P(J)$ for all $r, R>0$.

Indeed, $b(x, \nu):=\left(x^{(2)}-\lambda x^{(1)}, Z(x, \nu)-\lambda x^{(2)}\right)$ satisfies

$$
2\left\langle b\left(x_{1}, \nu_{1}\right)-b\left(x_{2}, \nu_{2}\right), x_{1}-x_{2}\right\rangle
$$




$$
\begin{aligned}
& \leq-2 \lambda\left|x_{1}^{(1)}-x_{2}^{(1)}\right|^{2}-2\left(\lambda-\alpha_{2}\right)\left|x_{1}^{(2)}-x_{2}^{(2)}\right|^{2} \\
& \quad+2\left|x_{1}^{(2)}-x_{2}^{(2)}\right|\left\{\left(1+\alpha_{1}\right)\left|x_{1}^{(1)}-x_{2}^{(1)}\right|+\alpha_{3} \mathbb{W}_{2}\left(\nu_{1}, \nu_{2}\right)\right\} \\
& \leq \alpha_{3} s \mathbb{W}_{2}\left(\nu_{1}, \nu_{2}\right)^{2}-\left\{2 \lambda-\delta\left(1+\alpha_{1}\right)\right\}\left|x_{1}^{(1)}-x_{2}^{(1)}\right|^{2} \\
& \quad-\left\{2 \lambda-2 \alpha_{2}-\delta^{-1}\left(1+\alpha_{1}\right)-\alpha_{3} s^{-1}\right\}\left|x_{1}^{(2)}-x_{2}^{(2)}\right|^{2}, \quad s, \delta>0
\end{aligned}
$$

for all $x_{1}, x_{2} \in \mathbb{R}^{2 m}$ and $\nu_{1}, \nu_{2} \in \mathscr{P}_{2}\left(\mathbb{R}^{2 m}\right)$. Taking

$$
\delta=\frac{2 \alpha_{2}+\alpha_{3} s^{-1}+\sqrt{4\left(1+\alpha_{1}\right)^{2}+\left(2 \alpha_{2}+\alpha_{3} r^{-1}\right)^{2}}}{2\left(1+\alpha_{1}\right)}
$$

such that $\delta\left(1+\alpha_{1}\right)=2 \alpha_{2}+\delta^{-1}\left(1+\alpha_{1}\right)+\alpha_{3} s^{-1}$, we see that $\left(H_{1}\right)$ holds for some $\kappa_{1}>\kappa_{2}$ provided $2 \lambda-\delta\left(1+\alpha_{1}\right)>\alpha_{3} s$ for some $s>0$, i.e. (2.5) implies $\left(H_{1}\right)$. Moreover, it is easy to see that conditions in Remark 2.1(1) hold, see also [7, 19] for Harnack inequalities and gradeint estimates on stochastic Hamiltonian systems which also imply the strong Feller and $\bar{\mu}$-irreducibility of $\bar{P}_{t}$. Therefore, the claimed assertion follows from Theorem 2.1(1).

\subsection{Distribution dependent SPDE}

Consider the following distribution-dependent SPDE on a separable Hilbert space $\mathbb{H}$ :

$$
\mathrm{d} X(t)=\left\{A X(t)+b\left(X(t), \mathscr{L}_{X(t)}\right)\right\} \mathrm{d} t+\sigma\left(\mathscr{L}_{X(t)}\right) \mathrm{d} W(t)
$$

where $(A, \mathscr{D}(A))$ is a linear operator on $\mathbb{H}, b: \mathbb{H} \times \mathscr{P}_{2}(\mathbb{H}) \rightarrow \mathbb{H}$ and $\sigma: \mathscr{P}_{2}(\mathbb{H}) \rightarrow \mathbb{L}(\tilde{\mathbb{H}} ; \mathbb{H})$ are measurable, and $W(t)$ is the cylindrical Brwonian motion on $\tilde{\mathbb{H}}$. We make the following assumption.

$\left(H_{2}\right)(-A, \mathscr{D}(A))$ is self-adjoint with discrete spectrum $0<\lambda_{1} \leq \lambda_{2} \leq \cdots$ counting multiplicities such that $\sum_{i=1}^{\infty} \lambda_{i}^{\gamma-1}<\infty$ holds for some constant $\gamma \in(0,1)$.

Moreover, $b$ is Lipschitz continuous on $\mathbb{H} \times \mathscr{P}_{2}(\mathbb{H}), \sigma$ is bounded and there exist constants $\alpha_{1}, \alpha_{2} \geq 0$ with $\lambda_{1}>\alpha_{1}+\alpha_{2}$ such that

$$
2\langle x-y, b(x, \mu)-b(y, \nu)\rangle+\|\sigma(\mu)-\sigma(\nu)\|_{H S}^{2} \leq 2 \alpha_{1}|x-y|^{2}+2 \alpha_{2} \mathbb{W}_{2}(\mu, \nu)^{2}
$$

holds for all $x, y \in \mathbb{H}$ and $\mu, \nu \in \mathscr{P}_{2}(\mathbb{H})$.

According to Theorem 3.1 below, assumption $\left(H_{2}\right)$ implies that for any $X(0) \in L^{2}(\Omega \rightarrow$ $\left.\mathbb{H}, \mathscr{F}_{0}, \mathbb{P}\right)$, the equation (2.6) has a unique mild solution $X(t)$. As before we denote by $X^{\nu}(t)$ the solution with initial distribution $\nu \in \mathscr{P}_{2}(\mathbb{H})$, and write $P_{t}^{*} \nu=\mathscr{L}_{X^{\nu}(t)}$. Moreover, by Itô's formula and $\kappa:=\lambda_{1}-\left(\alpha_{1}+\alpha_{2}\right)>0$, it is easy to see that $P_{t}^{*}$ has a unique invariant probability measure $\bar{\mu} \in \mathscr{P}_{2}(\mathbb{H})$ and

EXPP

$$
\mathbb{W}_{2}\left(P_{t}^{*} \nu, \bar{\mu}\right) \leq \mathrm{e}^{-\kappa t} \mathbb{W}_{2}(\nu, \bar{\mu}), \quad t \geq 0
$$

Consider the reference SPDE

$$
\mathrm{d} \bar{X}(t)=\{A \bar{X}(t)+b(\bar{X}(t), \bar{\mu})\} \mathrm{d} t+\sigma(\bar{\mu}) \mathrm{d} W(t),
$$


which is again well-posed for any initial value $\bar{X}(0) \in L^{2}\left(\Omega \rightarrow \mathbb{H}, \mathscr{F}_{0}, \mathbb{P}\right)$. Let $J$ be the DonskerVaradhan level 2 entropy function for the Markov process $\bar{X}(t)$, see Section 3. For any $r, R>0$ let

$$
\mathscr{B}_{r, R}:=\left\{\nu \in \mathscr{P}(\mathbb{H}): \nu\left(\mathrm{e}^{|\cdot|^{r}}\right) \leq R\right\} .
$$

T02 Theorem 2.2. Assume $\left(H_{2}\right)$. If there exist constants $\varepsilon \in(0,1)$ and $c>0$ such that

$$
\left\langle(-A)^{\gamma-1} x, b(x, \mu)\right\rangle \leq c+\varepsilon\left|(-A)^{\frac{\gamma}{2}} x\right|^{2}, \quad x \in \mathscr{D}\left((-A)^{\frac{\gamma}{2}}\right),
$$

then $\left\{L_{t}^{\nu}\right\}_{\nu \in \mathscr{B}_{r, R}} \in \operatorname{LDP}_{u}(J)$ for all $r, R>0$. If moreover $\bar{P}_{t}$ is strong Feller and $\bar{\mu}$-irreducible for some $t>0$, then $\left\{L_{t}^{\nu}\right\}_{\nu \in \mathscr{B}_{r, R}} \in L D P(J)$ for all $r, R>0$.

Assumption $\left(\mathrm{H}_{2}\right)$ is standard to imply the well-posedness of (2.6) and the exponential convergence of $P_{t}^{*}$ in $\mathbb{W}_{2}$. Condition (2.8) is implied by

$$
\left|(-A)^{\frac{\gamma}{2}-1} b(x, \mu)\right| \leq \varepsilon^{\prime}\left|(-A)^{\frac{\gamma}{2}} x\right|+c^{\prime}, \quad x \in \mathscr{D}\left((-A)^{\frac{\gamma}{2}}\right)
$$

for some constants $\varepsilon^{\prime} \in(0,1)$ and $c^{\prime}>0$. In particular, (2.8) holds if $|b(x, \mu)| \leq c_{1}+c_{2}|x|$ for some constants $c_{1}>0$ and $c_{2} \in\left(0, \lambda_{1}\right)$. When $\sigma$ is invertible with bounded $\sigma^{-1}$ and $b(\cdot, \mu)$ is Lipschitz continuous, the dimension-free Harnack inequality established in [16, Theorem 3.4.1] implies the strong Feller property and $\bar{\mu}$-irreducibility of $\bar{P}_{t}$ for $t>0$, see [16, Theorem 1.4.1] for more properties implied by this type Harnack inequality. Therefore, by Theorem 2.2, in this case $\left(H_{2}\right)$ and (2.9) imply $\left\{L_{t}^{\nu}\right\}_{\nu \in \mathscr{B}_{r, R}} \in L D P(J)$ for all $r, R>0$. See Example 2.4 below for the case where $\sigma$ is non-invertible and $b$ is possibly path-dependent.

\subsection{Path-distribution dependent SPDE with additive noise}

Let $\tilde{\mathbb{H}}=\mathbb{H}$ and $\sigma \in \mathbb{L}(\mathbb{H})$. Then (1.1) becomes

$$
\mathrm{d} X(t)=\left\{A X(t)+b\left(X_{t}, \mathscr{L}_{X_{t}}\right)\right\} \mathrm{d} t+\sigma \mathrm{d} W(t)
$$

Below we consider this equation with $\sigma$ being invertible and non-invertible respectively.

\subsubsection{Invertible $\sigma$}

Since $\sigma$ is constant, we are able to establish LDP for $b(\xi, \cdot)$ being Lipshcitz continuous in $\mathbb{W}_{p}$ for some $p \geq 1$ rather than just for $p=2$ as in the last two results.

$\left(H_{3}\right) \quad \sigma \in \mathbb{L}(\mathbb{H})$ is constant and $(A, \mathscr{D}(A))$ satisfies the corresponding condition in $\left(H_{2}\right)$. Moreover, there exist constants $p \geq 1$ and $\alpha_{1}, \alpha_{2} \geq 0$ such that

$$
|b(\xi, \mu)-b(\eta, \nu)| \leq \alpha_{1}\|\xi-\eta\|_{\infty}+\alpha_{2} \mathbb{W}_{p}(\mu, \nu), \quad \xi, \eta \in \mathscr{C}, \mu, \nu \in \mathscr{P}_{p}(\mathscr{C}) .
$$

Obviously, $\left(H_{3}\right)$ implies assumption (A) in Theorem 3.1 below, so that for any $X_{0}^{\nu} \in$ $L^{p}\left(\Omega \rightarrow \mathscr{C}, \mathscr{F}_{0}, \mathbb{P}\right)$ with $\nu=\mathscr{L}_{X_{0}^{\nu}}$, the equation (1.1) has a unique mild segment solution $X_{t}^{\nu}$ with

$$
\mathbb{E}\left[\sup _{t \in[0, T]}\left\|X_{t}^{\nu}\right\|_{\infty}^{p}\right]<\infty, \quad T>0 .
$$


Let $P_{t}^{*} \nu=\mathscr{L}_{X_{t}^{\nu}}$ for $t \geq 0$ and $\nu \in \mathscr{P}_{p}(\mathscr{C})$.

When $P_{t}^{*}$ has a unique invariant probability measure $\bar{\mu} \in \mathscr{P}_{p}(\mathscr{C})$, we consider the reference functional SPDE

$$
\mathrm{d} \bar{X}(t)=\left\{A \bar{X}(t)+b\left(\bar{X}_{t}, \bar{\mu}\right)\right\} \mathrm{d} t+\sigma \mathrm{d} W(t) .
$$

By Theorem 3.1 below, this reference equation is well-posed for any initial value in $L^{p}(\Omega \rightarrow$ $\left.\mathscr{C}, \mathscr{F}_{0}, \mathbb{P}\right)$. For any $\varepsilon, R>0$, let

$$
\mathscr{I}_{\varepsilon, R}=\left\{\nu \in \mathscr{P}(\mathscr{C}): \nu\left(\mathrm{e}^{\varepsilon\|\cdot\|_{\infty}^{2}}\right) \leq R\right\} .
$$

TL1 Theorem 2.3. Assume $\left(H_{3}\right)$. Let $\theta \in\left[0, \lambda_{1}\right]$ such that

$$
\kappa_{p}:=\theta-\left(\alpha_{1}+\alpha_{2}\right) \mathrm{e}^{p \theta r_{0}}=\sup _{r \in\left[0, \lambda_{1}\right]}\left\{r-\left(\alpha_{1}+\alpha_{2}\right) \mathrm{e}^{p r r_{0}}\right\} .
$$

(1) For any $\nu_{1}, \nu_{2} \in \mathscr{P}_{p}(\mathscr{C})$,

$$
\mathbb{W}_{p}\left(P_{t}^{*} \nu_{1}, P_{t}^{*} \nu_{2}\right)^{p} \leq \mathrm{e}^{p \theta r_{0}-p \kappa_{p} t} \mathbb{W}_{p}\left(\nu_{1}, \nu_{2}\right)^{p}, \quad t \geq 0 .
$$

In particular, if $\kappa_{p}>0$, then $P_{t}^{*}$ has a unique invariant probability measure $\bar{\mu} \in \mathscr{P}_{p}(\mathscr{C})$ such that

$$
\mathbb{W}_{p}\left(P_{t}^{*} \nu, \bar{\mu}\right)^{p} \leq \mathrm{e}^{p \theta r_{0}-p \kappa_{p} t} \mathbb{W}_{p}(\nu, \bar{\mu})^{p}, \quad t \geq 0, \nu \in \mathscr{P}_{p}(\mathscr{C})
$$

(2) Let $\sigma$ be invertible. If $\kappa_{p}>0$ and $\sup _{s \in\left(0, \lambda_{1}\right]}\left(s-\alpha_{1} \mathrm{e}^{s r_{0}}\right)>0$, then $\left\{L_{t}^{\nu}\right\}_{\nu \in \mathscr{I}_{\varepsilon, R}} \in \operatorname{LDP}(J)$ for any $\varepsilon, R>0$, where $J$ is the Donsker-Varadhan level 2 entropy function for the Markov process $\bar{X}_{t}$ on $\mathscr{C}$.

Example 2.3. For a bounded domain $D \subset \mathbb{R}^{d}$, let $\mathbb{H}=L^{2}(D ; \mathrm{d} x)$ and $A=-(-\Delta)^{\alpha}$, where $\Delta$ is the Dirichlet Laplacian on $D$ and $\alpha>\frac{d}{2}$ is a constant. Let $\sigma=I$ be the identity operator on $\mathbb{H}$, and

$$
b(\xi, \mu)=b_{0}(\mu)+\alpha_{1} \int_{-r_{0}}^{0} \xi(r) \Theta(\mathrm{d} r), \quad(\xi, \mu) \in \mathscr{C} \times \mathscr{P}_{1}(\mathscr{C}),
$$

where $\alpha_{1} \geq 0$ is a constant, $\Theta$ is a signed measure on $\left[-r_{0}, 0\right]$ with total variation 1 (i.e. $\left.|\Theta|\left(\left[-r_{0}, 0\right]\right)=1\right)$, and $b_{0}$ satisfies

$$
\left|b_{0}(\mu)-b_{0}(\nu)\right| \leq \alpha_{2} \mathbb{W}_{1}(\mu, \nu), \quad \mu, \nu \in \mathscr{P}_{1}(\mathscr{C})
$$

for some constant $\alpha_{2} \geq 0$. Then $\left(H_{3}\right)$ holds for $p=1$, and as shown in he proof of Example 1.1 in [1] that

$$
\lambda_{1} \geq \lambda:=\frac{\left(d \pi^{2}\right)^{\alpha}}{R(D)^{2 \alpha}},
$$

where $R(D)$ is the diameter of $D$. Therefore, all assertions in Theorem 2.3 hold provided

$$
\sup _{r \in(0, \lambda]}\left\{r-\left(\alpha_{1}+\alpha_{2}\right) \mathrm{e}^{r r_{0}}\right\}>0 .
$$

In particular, under this condition $\left\{L_{t}^{\nu}\right\}_{\nu \in \mathscr{I}_{\varepsilon, R}} \in L D P(J)$ for any $\varepsilon, R>1$. 


\subsubsection{Non-invertible $\sigma$}

Let $\mathbb{H}=\mathbb{H}_{1} \times \mathbb{H}_{2}$ for two separable Hilbert spaces $\mathbb{H}_{1}$ and $\mathbb{H}_{2}$, and consider the following path-distribution dependent SPDE for $X(t)=\left(X^{(1)}(t), X^{(2)}(t)\right)$ on $\mathbb{H}$ :

$$
\left\{\begin{array}{l}
\mathrm{d} X^{(1)}(t)=\left\{A_{1} X^{(1)}(t)+B X^{(2)}(t)\right\} \mathrm{d} t \\
\mathrm{~d} X^{(2)}(t)=\left\{A_{2} X^{(2)}(t)+Z\left(X_{t}, \mathscr{L}_{X_{t}}\right)\right\} \mathrm{d} t+\sigma \mathrm{d} W(t)
\end{array}\right.
$$

where $\left(A_{i}, \mathscr{D}\left(A_{i}\right)\right)$ is a densely defined closed linear operator on $\mathbb{H}_{i}$ generating a $C_{0}$-semigroup $\mathrm{e}^{t A_{i}}(i=1,2), B \in \mathbb{L}\left(\mathbb{H}_{2} ; \mathbb{H}_{1}\right), Z: \mathscr{C} \mapsto \mathbb{H}_{2}$ is measurable, $\sigma \in \mathbb{L}\left(\mathbb{H}_{2}\right)$, and $W(t)$ is the cylindrical Wiener process on $\mathbb{H}_{2}$. Obviously, (2.14) can be reduced to (2.10) by taking $A=$ $\operatorname{diag}\left\{A_{1}, A_{2}\right\}$ and using $\operatorname{diag}\{0, \sigma\}$ replacing $\sigma$, i.e. (2.14) is a special case of (2.10) with noninvertible $\sigma$.

For any $\alpha>0$ and $p \geq 1$, define

$$
\mathbb{W}_{p, \alpha}\left(\nu_{1}, \nu_{2}\right):=\inf _{\pi \in \mathscr{C}\left(\nu_{1}, \nu_{2}\right)}\left(\int_{\mathscr{C} \times \mathscr{C}}\left(\alpha\left\|\xi_{1}^{(1)}-\xi_{2}^{(1)}\right\|_{\infty}+\left\|\xi_{1}^{(2)}-\xi_{2}^{(2)}\right\|_{\infty}\right)^{p} \pi\left(\mathrm{d} \xi_{1}, \mathrm{~d} \xi_{2}\right)\right)^{\frac{1}{p}}
$$

We assume

$\left(H_{4}\right) \quad$ Let $p \geq 1$ and $\alpha>0$.

$\left(H_{4}^{1}\right)\left(-A_{2}, \mathscr{D}\left(A_{2}\right)\right)$ is self-adjoint with discrete spectrum $0<\lambda_{1} \leq \lambda_{2} \leq \cdots$ counting multiplicities such that $\sum_{i=1}^{\infty} \lambda_{i}^{\gamma-1}<\infty$ for some $\gamma \in(0,1)$. Moreover, $A_{1} \leq \delta-\lambda_{1}$ for some constant $\delta \geq 0$; i.e., $\left\langle A_{1} x, x\right\rangle \leq\left(\delta-\lambda_{1}\right)|x|^{2}$ holds for all $x \in \mathscr{D}\left(A_{1}\right)$.

$\left(H_{4}^{2}\right)$ There exist constants $K_{1}, K_{2}>0$ such that

$$
\left|Z\left(\xi_{1}, \nu_{1}\right)-b\left(\xi_{2}, \nu_{2}\right)\right| \leq K_{1}\left\|\xi_{1}^{(1)}-\xi_{2}^{(1)}\right\|_{\infty}+K_{2}\left\|\xi_{1}^{(2)}-\xi_{2}^{(2)}\right\|_{\infty}+K_{3} \mathbb{W}_{p, \alpha}\left(\nu_{1}, \nu_{2}\right), \quad\left(\xi_{i}, \nu_{i}\right) \in \mathscr{C} \times \mathscr{P}_{p}(\mathscr{C}) .
$$

$\left(H_{4}^{3}\right) \sigma$ is invertible on $\mathbb{H}_{2}$, and there exists $A_{0} \in \mathbb{L}\left(\mathbb{H}_{1} ; \mathbb{H}_{1}\right)$ such that for any $t>0, B \mathrm{e}^{t A_{2}}=$ $\mathrm{e}^{t A_{1}} \mathrm{e}^{t A_{0}} B$ holds and

$$
Q_{t}:=\int_{0}^{t} \mathrm{e}^{s A_{0}} B B^{*} \mathrm{e}^{s A_{0}^{*}} \mathrm{~d} s
$$

is invertible on $\mathbb{H}_{1}$.

By Theorem 3.1 for $\mathbb{H}_{0}=\mathbb{H}_{2}$ and $\operatorname{diag}\{0, \sigma\}$ replacing $\sigma,\left(H_{4}\right)$ implies that for any $X_{0} \in$ $L^{p}\left(\Omega \rightarrow \mathscr{C}, \mathscr{F}_{0}, \mathbb{P}\right)$ this equation has a unique mild segment solution. Let $P_{t}^{*} \nu=\mathscr{L}_{X_{t}}$ for $\mathscr{L}_{X_{0}}=\nu \in \mathscr{P}_{p}(\mathscr{C})$.

T03 Theorem 2.4. Assume $\left(H_{4}\right)$ for some constants $p \geq 1$ and $\alpha>0$ satisfying

$$
\alpha \leq \alpha^{\prime}:=\frac{1}{2\|B\|}\left\{\delta-K_{2}+\sqrt{\left(\delta-K_{2}\right)^{2}+4 K_{1}\|B\|}\right\},
$$

where $\|\cdot\|$ is the operator norm. If

$$
\inf _{s \in\left(0, \lambda_{1}\right]} s \mathrm{e}^{-s r_{0}}>K_{2}+\alpha^{\prime}\|B\|+K_{3},
$$


then $P_{t}^{*}$ has a unique invariant probability measure $\bar{\mu}$ such that

$$
\mathbb{W}_{p}\left(P_{t}^{*} \nu, \bar{\mu}\right)^{2} \leq c_{1} \mathrm{e}^{-c_{2} t} \mathbb{W}_{p}(\nu, \bar{\mu}), \quad \nu \in \mathscr{P}_{p}(\mathscr{C}), t \geq 0
$$

holds for some constants $c_{1}, c_{2}>0$, and $\left\{L_{t}^{\nu}\right\}_{\nu \in \mathscr{I}_{\varepsilon, R}} \in L D P(J)$ for any $\varepsilon, R>1$, where $J$ is the Donsker-Varadhan level 2 entropy function for the associated reference equation for $\bar{X}(t)$.

Example 2.4. Consider the following equation for $X(t)=\left(X^{(1)}(t), X^{(2)}(t)\right)$ on $\mathbb{H}=\mathbb{H}_{0} \times \mathbb{H}_{0}$ for a separable Hilbert space $\mathbb{H}_{0}$ :

$$
\left\{\begin{array}{l}
\mathrm{d} X^{(1)}(t)=\left\{\alpha_{1} X^{(2)}(t)-\lambda_{1} X^{(1)}(t)\right\} \mathrm{d} t \\
\mathrm{~d} X^{(2)}(t)=\left\{Z\left(X(t), \mathscr{L}_{X(t)}\right)-A X^{(2)}(t)\right\} \mathrm{d} t+\mathrm{d} W(t),
\end{array}\right.
$$

where $\alpha_{1} \in \mathbb{R} \backslash\{0\}, W(t)$ is the cylindrical Brownian motion on $\mathbb{H}_{0}, A$ is a self-adjoint operator on $\mathbb{H}_{0}$ with discrete spectrum such that all eigenvalues $0<\lambda_{1} \leq \ll_{2} \leq \cdots$ counting multiplicities satisfy

$$
\sum_{i=1}^{\infty} \lambda_{i}^{\gamma-1}<\infty
$$

for some $\gamma \in(0,1)$, and $Z$ satisfies

$$
\left|Z\left(\xi_{1}, \nu_{1}\right)-Z\left(\xi_{2}, \nu_{2}\right)\right| \leq \alpha_{2}\left\|\xi_{1}-\xi_{2}\right\|_{\infty}+\alpha_{3} \mathbb{W}_{2}\left(\nu_{1}, \nu_{2}\right), \quad\left(\xi_{i}, \nu_{i}\right) \in \mathscr{C} \times \mathscr{P}_{2}(\mathscr{C}), i=1,2 .
$$

Let

$$
\alpha=\frac{1}{2 \alpha_{1}}\left(\sqrt{\alpha_{2}^{2}+4 \alpha_{1} \alpha_{2}}-\alpha_{2}\right) .
$$

Then $P_{t}^{*}$ has a unique invariant probability measure $\bar{\mu} \in \mathscr{P}_{2}(\mathscr{C})$, and $\left\{L_{t}^{\nu}\right\}_{\nu \in \mathscr{I}_{R, q}} \in L D P(J)$ for any $R, q>1$ if

$$
\inf _{s \in\left[0, \lambda_{1}\right]} s \mathrm{e}^{-s r_{0}}>\alpha_{2}+\alpha_{1} \alpha+\frac{\alpha_{3}}{1 \wedge \alpha} .
$$

Indeed, it is easy to see that assumption $\left(H_{4}\right)$ holds for $p=2, \delta=0,\|B\|=\alpha_{1}, K_{1}=K_{2}=\alpha_{2}$ and $K_{3}=\frac{\alpha_{3}}{1 \wedge \alpha}$. So, we have $\alpha=\alpha^{\prime}$ and (2.18) is equivalent to (2.16). Then the desired assertion follows from Theorem 2.4.

\section{Preparations}

In this part, we investigate path-distribution dependent SPDEs and recall some facts on Donsker-Varadhan LDP for Markov processes.

\subsection{Path-distribution dependent SPDEs}

Consider the following path-distribution dependent SPDE on $\mathbb{H}$ :

$$
\mathrm{d} X(t)=\left\{A X(t)+b_{t}\left(X_{t}, \mathscr{L}_{X_{t}}\right)\right\} \mathrm{d} t+\sigma_{t}\left(X_{t}, \mathscr{L}_{X_{t}}\right) \mathrm{d} W(t),
$$


where $(A, \mathscr{D}(A))$ is a negative self-adjoint operator on $\mathbb{H}$, and

$$
b:[0, \infty) \times \mathscr{C} \times \mathscr{P}(\mathscr{C}) \rightarrow \mathbb{H}, \quad \sigma:[0, \infty) \times \mathscr{C} \times \mathscr{P}(\mathscr{C}) \rightarrow \mathbb{L}(\mathbb{H} ; \tilde{\mathbb{H}})
$$

are measurable, and $W(t)$ is the cylindrical Brownian motion on $\tilde{\mathbb{H}}$.

DF Definition 3.1. An adapted continuous process $\left(X_{t}\right)_{t \geq 0}$ on $\mathscr{C}$ is called a mild segment (or functional) solution of (3.1), if

$$
\mathbb{E} \int_{0}^{t}\left\{\left|\mathrm{e}^{(t-s) A} b_{s}\left(X_{s}, \mathscr{L}_{X_{s}}\right)\right|+\left\|\mathrm{e}^{(t-s) A} \sigma_{s}\left(X_{s}, \mathscr{L}_{X_{s}}\right)\right\|_{H S}^{2}\right\} \mathrm{d} s<\infty, \quad t \geq 0,
$$

and the process $X(t):=X_{t}(0)$ satisfies $\mathbb{P}$-a.s.

$$
X(t)=\mathrm{e}^{A t} X(0)+\int_{0}^{t} \mathrm{e}^{(t-s) A} b_{s}\left(X_{s}, \mathscr{L}_{X_{s}}\right) \mathrm{d} s+\int_{0}^{t} \mathrm{e}^{(t-s) A} \sigma_{s}\left(X_{s}, \mathscr{L}_{X_{s}}\right) \mathrm{d} W(s), \quad t \geq 0 .
$$

In this case, we call $(X(t))_{t \geq 0}$ a mild solution of (3.1) with initial value $X_{0}$.

To ensure the existence and uniqueness of mild solutions with $X_{0} \in L^{p}\left(\Omega \rightarrow \mathscr{C}, \mathscr{F}_{0}, \mathbb{P}\right)$ for some $p>0$, we make the following assumption.

(A) Let $p \in(0, \infty)$. There exists a subspace $\mathbb{H}_{0}$ of $\mathbb{H}$ such that $\sigma(\xi, \nu) \tilde{\mathbb{H}} \subset \mathbb{H}_{0}$ for any $(\xi, \nu) \in \mathscr{C} \times \mathscr{P}(\mathscr{C})$, and the orthogonal projection $\pi_{0}: \mathbb{H} \rightarrow \mathbb{H}_{0}$ satisfies $A \pi_{0}=\pi_{0} A$ on $\mathscr{D}(A)$. Moreover, there exist $\gamma \in(0,1)$ and $1 \leq K \in L_{l o c}^{1}([0, \infty) \rightarrow[0, \infty))$ such that $\left(A_{1}\right) \int_{0}^{t} s^{-\gamma}\left\|\mathrm{e}^{s A} \pi_{0}\right\|_{H S}^{2} \mathrm{~d} s<\infty, t \in(0, \infty)$.

$\left(A_{2}\right)$ There exists $p_{0}>2$ such that for any $t \geq 0, \xi, \eta \in \mathscr{C}$ and $\mu, \nu \in \mathscr{P}_{p}(\mathscr{C})$,

$$
\begin{aligned}
& \left|b_{t}(\xi, \mu)-b_{t}(\eta, \nu)\right| \leq K(t)\left(\|\xi-\eta\|_{\infty}+\mathbb{W}_{p}(\mu, \nu)\right), \\
& \left\|\sigma_{t}(\xi, \mu)-\sigma_{t}(\eta, \nu)\right\|^{p} \leq K(t)^{1 \wedge \frac{p}{p_{0}}}\left(\|\xi-\eta\|_{\infty}^{p}+\mathbb{W}_{p}(\mu, \nu)^{p}\right) .
\end{aligned}
$$

$\left(A_{3}\right)\left|b_{t}\left(0, \delta_{0}\right)\right|+\left\|\sigma_{t}\left(0, \delta_{0}\right)\right\|^{p \vee p_{0}} \leq K(t), \quad t \geq 0$.

In many references $\left(A_{1}\right)$ is replaced by $\int_{0}^{t} s^{-\gamma}\left\|\mathrm{e}^{A s}\right\|_{H S}^{2} \mathrm{~d} s<\infty$, see for instance [3]. The present weaker version allows us to cover more examples with degenerate noise.

Remark 3.1. By (A) we have $\mathrm{e}^{A(t-s)} \sigma_{s}=\mathrm{e}^{A(t-s)} \pi_{0} \sigma_{s}$, so that using $\mathrm{e}^{A(t-s)} \pi_{0}$ to replace the semigroup $S(t-s)$ in the proof of [3], Proposition 7.9], if $\Phi(s)$ is an adapted process on $\mathbb{L}(\mathbb{H} ; \tilde{\mathbb{H}})$ such that $\mathbb{E} \int_{0}^{t}\|\Phi(s)\|^{q} \mathrm{~d} s<\infty$ for some $q>2$, then

$$
W_{\Phi}(t):=\int_{0}^{t} \mathrm{e}^{A(t-s)} \pi_{0} \Phi(s) \mathrm{d} W(s), \quad t \geq 0
$$

is an adapted continuous process on $\mathbb{H}$ such that

$$
\mathbb{E}\left[\left|\inf _{s \in[0, t]} \int_{0}^{t} \mathrm{e}^{A(s-r)} \pi_{0} \Phi(r) \mathrm{d} W(r)\right|^{q}\right] \leq c \mathbb{E} \int_{0}^{t}\|\Phi(s)\|^{q} \mathrm{~d} s
$$

holds for some constant $c>0$. 
EXU Theorem 3.1. Assume (A) and let $X_{0} \in L^{p}\left(\Omega \rightarrow \mathscr{C}, \mathscr{F}_{0}, \mathbb{P}\right)$. Then (3.1) has a unique mild segment solution $\left\{X_{t}\right\}_{t \geq 0}$ starting at $X_{0}$ with

$$
\mathbb{E}\left[\sup _{t \in[0, T]}\left\|X_{t}\right\|_{\infty}^{p}\right]<\infty, \quad T \in(0, \infty)
$$

provided one of following conditions holds:

(1) $p>2$.

(2) $p \in(0,2]$ and $\sigma_{s}(\xi, \mu)$ does not depend on $\xi$.

(3) $p=2$ and for any $s \geq 0, \xi, \eta \in \mathscr{C}$ and $\mu, \nu \in \mathscr{P}_{2}(\mathscr{C})$,

$$
\left\|\sigma_{s}(\xi, \mu)-\sigma_{s}(\eta, \nu)\right\|_{H S}^{2} \leq K(s)\left\{\|\xi-\eta\|_{\infty}^{2}+\mathbb{W}_{2}(\mu, \nu)^{2}\right\} .
$$

Proof. We consider cases (1)-(3) respectively.

Proof for Case (1). Let $p>2$.

The existence. We adopt an iteration argument as in [18]. It suffices to prove that for any fixed $T>0$, the SPDE has a unique mild segment solution up to time $T$ satisfying

$$
\mathbb{E}\left[\sup _{t \in[0, T]}\left\|X_{t}\right\|_{\infty}^{p}\right]<\infty
$$

(1a) We first consider the case that $X_{0}$ is bounded. Let $X_{t}^{0}=X_{0}$ and $\mu_{t}^{0}=\mathscr{L}_{X_{t}^{0}}$ for $t \geq 0$. By Remark 3.1,

$$
X^{1}(t):=\mathrm{e}^{A t} X(0)+\int_{0}^{t} \mathrm{e}^{(t-s) A} b_{s}\left(X_{s}^{0}, \mu_{s}^{0}\right) \mathrm{d} s+\int_{0}^{t} \mathrm{e}^{(t-s) A} \sigma_{s}\left(X_{s}^{0}, \mu_{s}^{0}\right) \mathrm{d} W(s), \quad t \geq 0,
$$

is an adapted continuous process on $\mathbb{H}$ such that

$$
\mathbb{E}\left[\sup _{t \in[0, T]}\left\|X_{t}^{1}\right\|_{\infty}^{q}\right]<\infty, \quad q>0
$$

where $X_{t}^{1}(r):=X^{1}(t+r) 1_{\{t+r \geq 0\}}+X_{0}(t+r) 1_{\{t+r<0\}}$.

Now, assume that for some $n \geq 1$ we have constructed a continuous adapted process $\left\{X_{t}^{n}\right\}_{t \in[0, T]}$ on $\mathscr{C}$ with $X_{0}^{n}=X_{0}$ and

$$
\mathbb{E}\left[\sup _{t \in[0, T]}\left\|X_{t}^{n}\right\|_{\infty}^{q}\right]<\infty, \quad q>0
$$

By Remark 3.1,

$$
X^{n+1}(t):=\mathrm{e}^{A t} X(0)+\int_{0}^{t} \mathrm{e}^{(t-s) A} b_{s}\left(X_{s}^{n}, \mu_{s}^{n}\right) \mathrm{d} s+\int_{0}^{t} \mathrm{e}^{(t-s) A} \sigma_{s}\left(X_{s}^{n}, \mu_{s}^{n}\right) \mathrm{d} W(s), \quad t \in[0, T]
$$


for $\mu_{s}^{n}:=\mathscr{L}_{X_{s}^{n}}$ is an adapted continuous process on $\mathbb{H}$, and the segment process $X_{t}^{n+1}$ given by

$$
X_{t}^{n+1}(r):=X^{n+1}(t+r) 1_{\{t+r \geq 0\}}+X_{0}(t+r) 1_{\{t+r<0\}} \text { for } r \in\left[-r_{0}, 0\right], \quad t \geq 0
$$

satisfies

$$
\mathbb{E}\left[\sup _{t \in[0, T]}\left\|X_{t}^{n+1}\right\|_{\infty}^{q}\right]<\infty, \quad q>0
$$

It suffices to find a constant $t_{0}>0$ independent of $X_{0}$ such that $\left\{X_{\left[0, t_{0}\right]}^{n}\right\}_{n \geq 1}$ is a Cauchy sequence in $L^{p}\left(\Omega \rightarrow C\left(\left[0, t_{0}\right] ; \mathscr{C}\right), \mathbb{P}\right)$. This together with assumption $(\mathbf{A})$ imply that the limit $X_{\left[0, t_{0}\right]}:=\lim _{n \rightarrow \infty} X_{\left[0, t_{0}\right]}^{n}$ gives rise to a mild segment solution of (3.1) up to time $t_{0}$. By repeating the procedure with initial time $i t_{0}$ and initial value $X_{i t_{0}}$ for $i \geq 1$, in finite many steps we may construct a mild segment solution of (3.1) up to time $T$, such that (3.2) holds.

For any $n \geq 1$, by (3.4), (3.5) and assumption (A) we have

$* \mathrm{RR}$

$$
\begin{aligned}
& \psi_{n}(t):=\sup _{s \in[0, t]}\left\|X_{s}^{n+1}-X_{s}^{n}\right\|_{\infty}=\sup _{s \in[0, t]}\left|X^{n+1}(s)-X^{n}(s)\right| \\
& \leq \int_{0}^{t} K(s)\left\{\left\|X_{s}^{n}-X_{s}^{n-1}\right\|_{\infty}+\mathbb{W}_{p}\left(\mu_{s}^{n}, \mu_{s}^{n-1}\right)\right\} \mathrm{d} s+\sup _{s \in[0, t]}\left|\int_{0}^{s} \mathrm{e}^{A(s-r)} \Phi_{n}(r) \mathrm{d} W(r)\right|,
\end{aligned}
$$

where $\Phi_{n}(r):=\sigma_{s}\left(X_{r}^{n}, \mu_{r}^{n}\right)-\sigma_{s}\left(X_{r}^{n-1}, \mu_{r}^{n-1}\right)$ satisfies

$$
\left\|\Phi_{n}(r)\right\|^{p} \leq K(r)\left\{\left\|X_{r}^{n}-X_{r}^{n-1}\right\|_{\infty}^{p}+\mathbb{W}_{p}\left(\mu_{r}^{n}, \mu_{r}^{n-1}\right)^{p}\right\} .
$$

Combining this with $\mathbb{W}_{p}\left(\mu_{r}^{n}, \mu_{r}^{n-1}\right)^{p} \leq \mathbb{E}\left\|X_{r}^{n}-X_{r}^{n-1}\right\|_{\infty}^{p}$, and noting that Remark 3.1 implies

$$
\mathbb{E}\left[\sup _{s \in[0, t]}\left|\int_{0}^{s} \mathrm{e}^{A(s-r)} \Phi_{n}(r) \mathrm{d} W(r)\right|^{p}\right] \leq c \mathbb{E} \int_{0}^{t}\left\|\Phi_{n}(s)\right\|^{p} \mathrm{~d} s
$$

for some constant $c>0$, we find constants $C_{1}, C_{2}>0$ such that

$$
\begin{aligned}
\mathbb{E}\left[\psi_{n}(t)^{p}\right] \leq & C_{1} \mathbb{E}\left(\int_{0}^{t} K(s)\left\{\psi_{n-1}(s)+\mathbb{W}_{p}\left(\mu_{s}^{n}, \mu_{s}^{n-1}\right) \mathrm{d} s\right)^{p}\right. \\
& +C_{1} \mathbb{E} \int_{0}^{t} K(s)\left\{\left\|X_{s}^{n}-X_{s}^{n-1}\right\|_{\infty}^{p}+\mathbb{W}_{p}\left(\mu_{s}^{n}, \mu_{s}^{n-1}\right)^{p}\right\} \mathrm{d} s \\
\leq & C_{2} \varepsilon(t) \mathbb{E}\left[\psi_{n-1}^{p}(t)\right], \quad \varepsilon(t):=\left(\int_{0}^{t} K(s) \mathrm{d} s\right)^{p}+\int_{0}^{t} K(s) \mathrm{d} s .
\end{aligned}
$$

Taking $t_{0} \in(0, T]$ such that $C_{2} \varepsilon(t) \leq \frac{1}{2}$, we obtain

$$
\mathbb{E}\left[\psi_{n}^{p}\left(t_{0}\right)\right] \leq 2^{-n} \mathbb{E}\left[\psi_{0}^{p}\left(t_{0}\right)\right]<\infty, \quad n \geq 1 .
$$

Thus, $\left\{X_{\left[0, t_{0}\right]}^{n}\right\}_{n \geq 1}$ is a Cauchy sequence in $L^{p}\left(\Omega \rightarrow C\left(\left[0, t_{0}\right] ; \mathscr{C}\right), \mathbb{P}\right)$ as desired.

(1b) In general, for $X_{0} \in L^{p}\left(\Omega \rightarrow \mathscr{C}, \mathscr{F}_{0}, \mathbb{P}\right)$ and $N \in \mathbb{N}$ let $X_{0}^{(N)}=X_{0} 1_{\left\{\left\|X_{0}\right\|_{\infty} \leq N\right\}}$. By (1a), for any $N \geq 1$ we have constructed a mild segment solution $\left(X_{t}^{(N)}\right)_{t \in[0, T]}$ for (3.1) satisfying (3.2) with initial value $X_{0}^{(N)}$ :

$X^{N}(t):=\mathrm{e}^{A t} X^{(N)}(0)+\int_{0}^{t} \mathrm{e}^{(t-s) A} b_{s}\left(X_{s}^{(N)}, \mu_{s}^{(N)}\right) \mathrm{d} s+\int_{0}^{t} \mathrm{e}^{(t-s) A} \sigma_{s}\left(X_{s}^{(N)}, \mu_{s}^{(N)}\right) \mathrm{d} W(s), \quad t \in[0, T]$, 
where $\mu_{s}^{(N)}=\mathscr{L}_{X_{s}^{(N)}}$. By the above argument for $X^{(N)}(t)-X^{(M)}(t)$ instead of $X^{n+1}(t)-X^{n}(t)$, we find a constant $C>0$ such that for any $N, M \geq 1$, the process

$$
\psi_{N, M}(t):=\sup _{s \in[0, t]}\left\|X_{s}^{(N)}-X_{s}^{(M)}\right\|_{\infty}^{p}, \quad t \in[0, T]
$$

satisfies

$$
\mathbb{E}\left[\psi_{N, M}(t)\right] \leq C \mathbb{E}\left[\left\|X_{0}\right\|_{\infty}^{p} 1_{\left\{\left\|X_{0}\right\|_{\infty}>N \wedge M\right\}}\right]+C \varepsilon(t) \mathbb{E}\left[\psi_{N, M}(t)\right], \quad t \in[0, T] .
$$

Taking $t_{0} \in(0, T]$ such that $C \varepsilon\left(t_{0}\right) \leq \frac{1}{2}$, we obtain

$$
\mathbb{E}\left[\psi_{N, M}\left(t_{0}\right)\right] \leq 2 C \mathbb{E}\left[\left\|X_{0}\right\|_{\infty}^{p} 1_{\left\{\left\|X_{0}\right\|_{\infty}>N \wedge M\right\}}\right], \quad N, M \geq 1
$$

so that, $\left\{X_{\left[0, t_{0}\right]}^{(N)}\right\}_{N \geq 1}$ is a Cauchy sequence in $L^{p}\left(\Omega \rightarrow C\left(\left[0, t_{0}\right] ; \mathscr{C}\right), \mathbb{P}\right)$, and it is easy to see that its limit as $N \rightarrow \infty$ is a solution of (3.1) up to time $t_{0}$. As explained before that by repeating the procedure we construct a mild segment solution of (3.1) up to time $T$ satisfying (3.2).

The uniqueness. Let $X(t)$ and $Y(t)$ be two mild segment solutions with initial value $X_{0}$ satisfying

$$
\mathbb{E}\left[\sup _{t \in[0, T]}\left(\left\|X_{t}\right\|_{\infty}^{p}+\left\|Y_{t}\right\|_{\infty}^{p}\right)\right]<\infty .
$$

Similarly to (3.8) we have

$$
\mathbb{E}\left[\sup _{s \in[0, t]}\left\|X_{s}-Y_{s}\right\|_{\infty}^{p}\right] \leq C \varepsilon(t) \mathbb{E}\left[\sup _{s \in[0, t]}\left\|X_{s}-Y_{s}\right\|_{\infty}^{p}\right], \quad t \in[0, T] .
$$

This implies $X_{t}=Y_{t}$ up to time $t_{0} \in(0, T]$ such that $C \varepsilon\left(t_{0}\right)<1$. Since this $t_{0}$ does not depend on the initial value, repeating the same argument leads to $X_{t}=Y_{t}$ for all $t \in[0, T]$.

Proof for Case (2). Let $p \in(0,2]$. Again we first assume that $X_{0}$ is bounded and let $X^{n}, \mu^{n}, \psi_{n}$ be defined in step (1a). Since $\sigma_{s}(\xi, \mu)$ does not depend on $\xi$ and $K(s) \geq 1$, by $\left(A_{2}\right), \Phi_{n}(s)$ in (3.6) satisfies

$$
\left\|\Phi_{n}(s)\right\|^{p_{0}} \leq K(s) \mathbb{W}_{p}\left(\mu_{s}^{n}, \mu_{s}^{n-1}\right)^{p_{0}} .
$$

Combining this with Remark 3.1 for $q=p_{0}>2$, and using $\mathbb{W}_{p}\left(\mu_{s}^{n}, \mu_{s}^{n-1}\right)^{p} \leq \mathbb{E}\left\|X_{s}^{n}-X_{s}^{n-1}\right\|_{\infty}^{p}$, we find a constant $C_{1}>0$ such that

$$
\begin{aligned}
\mathbb{E}\left[\psi_{n}^{p}(t)\right] & \leq C_{1}\left(\mathbb{E} \int_{0}^{t} K(s) \psi_{n-1}(s) \mathrm{d} s\right)^{p}+C_{1}\left(\int_{0}^{t} K(s) \mathbb{W}_{p}\left(\mu_{s}^{n}, \mu_{s}^{n-1}\right)^{p_{0}} \mathrm{~d} s\right)^{\frac{p}{p_{0}}} \\
& \leq C_{1} \delta(t) \mathbb{E}\left[\psi_{n-1}^{p}(t)\right], \quad t \in[0, T], n \geq 1
\end{aligned}
$$

holds for $\delta(t):=\left(\int_{0}^{t} K(s) \mathrm{d} s\right)^{p}+\left(\int_{0}^{t} K(s) \mathrm{d} s\right)^{\frac{p}{p_{0}}}$. Then the remainder of the proof, including the existence and uniqueness for bounded $X_{0}$, and the extension to general $X_{0} \in L^{p}\left(\Omega \rightarrow \mathscr{C}, \mathscr{F}_{0}, \mathbb{P}\right)$, is similar to that in Case (1).

Proof for Case (3). Let $p=2$. As explained above we only consider bounded $X_{0}$. In this case, 
let $X^{n}, \mu^{n}, \psi_{n}$ be defined in step (1a). By (A) and Itô's formula to $\left|X^{n+1}(t)-X^{n}(t)\right|^{2}$, we find a constant $c>0$ such that

$$
\begin{aligned}
& \mathrm{d}\left|X^{n+1}(t)-X^{n}(t)\right|^{2} \\
& \leq c K(t)\left\{\left|X^{n+1}(t)-X^{n}(t)\right|^{2}+\left\|X_{t}^{n}-X_{t}^{n-1}\right\|_{\infty}^{2}+\mathbb{W}_{2}\left(\mu_{t}^{n}, \mu_{t}^{n-1}\right)^{2}\right\} \mathrm{d} t+\mathrm{d} M^{n}(t),
\end{aligned}
$$

where

$$
M^{n}(t):=2 \int_{0}^{t}\left\langle X^{n+1}(s)-X^{n}(s),\left\{\sigma_{s}\left(X_{s}^{n}, \mu_{s}^{n}\right)-\sigma_{s}\left(X_{s}^{n-1}, \mu_{s}^{n-1}\right)\right\} \mathrm{d} W(s)\right\rangle
$$

satisfies

$$
\mathrm{d}\left\langle M^{n}(t)\right\rangle \leq 4 K(t)\left|X^{n+1}(t)-X^{n}(t)\right|^{2}\left\{\left\|X_{t}^{n}-X_{t}^{n-1}\right\|_{\infty}^{2}+\mathbb{W}_{2}\left(\mu_{t}^{n}, \mu_{t}^{n-1}\right)^{2}\right\} \mathrm{d} t .
$$

Obviously, (3.9) implies

$$
\begin{aligned}
& \left|X^{n+1}(t)-X^{n}(t)\right|^{2} \mathrm{e}^{-c \int_{0}^{t} K(s) d s} \\
& \leq \int_{0}^{t} K(s) \mathrm{e}^{-c \int_{0}^{s} K(r) \mathrm{d} r}\left(\left\|X_{s}^{n}-X_{s}^{n-1}\right\|_{\infty}^{2}+\mathbb{W}_{2}\left(\mu_{s}^{n}, \mu_{s}^{n-1}\right)^{2}\right) \mathrm{d} s+\int_{0}^{t} \mathrm{e}^{-\int_{0}^{s} c K(r) \mathrm{d} r} \mathrm{~d} M^{n}(s)
\end{aligned}
$$

for $t \in[0, T]$. Therefore, by the BDG inequality, there exist constants $C_{1}, C_{2}>0$ depending only on $T$ such that

$$
\begin{aligned}
& \mathbb{E}\left[\psi_{n}^{2}(t)\right] \leq \mathrm{e}^{c \int_{0}^{T} K(s) \mathrm{d} s} \mathbb{E}\left[\sup _{s \in[0, t]}\left|X^{n+1}(s)-X^{n}(s)\right|^{2} \mathrm{e}^{\left.-c \int_{0}^{s} K(r) d r\right]}\right. \\
& \leq C_{1} \int_{0}^{t} K(s)\left\{\mathbb{E}\left[\psi_{n-1}^{2}(s)\right]+\mathbb{W}_{2}\left(\mu_{s}^{n}, \mu_{s}^{n-1}\right)^{2}\right\} \mathrm{d} s \\
& \quad+C_{1} \mathbb{E}\left[\left(\int_{0}^{t} K(s) \psi_{n}^{2}(s)\left\{\psi_{n-1}^{2}(s)+\mathbb{W}_{2}\left(\mu_{s}^{n}, \mu_{s}^{n-1}\right)^{2}\right\} \mathrm{d} s\right)^{\frac{1}{2}}\right] \\
& \leq \frac{1}{2} \mathbb{E}\left[\psi_{n}^{2}(t)\right]+C_{2} \int_{0}^{t} K(s)\left\{\mathbb{E}\left[\psi_{n-1}^{2}(s)\right]+\mathbb{W}_{2}\left(\mu_{s}^{n}, \mu_{s}^{n-1}\right)^{2}\right\} \mathrm{d} s, \quad t \in[0, T] .
\end{aligned}
$$

Noting that $\mathbb{W}_{2}\left(\mu_{s}^{n}, \mu_{s}^{n-1}\right)^{2} \leq \mathbb{E}\left[\psi_{n-1}^{2}(s)\right]$, this implies

$$
\mathbb{E}\left[\psi_{n}^{2}(t)\right] \leq 4 C_{2} \int_{0}^{t} K(s) \mathbb{E}\left[\psi_{n-1}^{2}(s)\right] \mathrm{d} s \leq 4 C_{2} \mathbb{E}\left[\psi_{n-1}^{2}(t)\right] \int_{0}^{t} K(s) \mathrm{d} s, \quad t \in[0, T], n \geq 1 .
$$

Taking $t_{0} \in(0, T]$ such that $4 C_{2} \int_{0}^{t_{0}} K(s) \mathrm{d} s \leq \frac{1}{2}$, we obtain

$$
\mathbb{E}\left[\psi_{n}^{2}\left(t_{0}\right)\right] \leq 2^{-(n-1)} \mathbb{E}\left[\psi_{0}^{2}\left(t_{0}\right)\right]<\infty, \quad n \geq 1
$$

Thus, $\left\{X_{\left[0, t_{0}\right]}^{n}\right\}_{n \geq 1}$ is a Cauchy sequence in $L^{2}\left(\Omega \rightarrow C\left(\left[0, t_{0}\right] ; \mathscr{C}\right), \mathbb{P}\right)$ as desired. The remainder of the proof is similar to that in Case (1). 


\subsection{Donsker-Varadhan LDP for Markov processes}

We first introduce the rate function, i.e. the Donsker-Varadhan level 2 entropy function for continuous Markov processes on a Polish space E.

Consider the path space

$$
\mathbf{C}_{E}:=C([0, \infty) \rightarrow E)=\{w:[0, \infty) \ni t \mapsto w(t) \in E \text { is continuous }\} .
$$

Let $\mathscr{P}\left(\mathbf{C}_{E}\right)$ be the set of all probability measures on $\mathbf{C}_{E}$, and $\mathscr{P}^{s}\left(\mathbf{C}_{E}\right)$ the set of all stationary (i.e. time-shift-invariant) elements in $\mathscr{P}\left(\mathbf{C}_{E}\right)$. For any $Q \in \mathscr{P}^{s}\left(\mathbf{C}_{E}\right)$, let $\bar{Q}$ be the unique stationary probability measure on $\overline{\mathbf{C}}_{E}:=C(\mathbb{R} \rightarrow E)$ such that

$$
\bar{Q}\left(\left\{w \in \overline{\mathbf{C}}_{E}: w\left(t_{i}\right) \in A_{i}, 1 \leq i \leq n\right\}\right)=Q\left(\left\{w \in \mathbf{C}_{E}: w\left(t_{i}+s\right) \in A_{i}, 1 \leq i \leq n\right\}\right)
$$

holds for any $n \geq 1,-\infty<t_{1}<t_{2}<\cdots<t_{n}<\infty, s \geq-t_{1}$, and $\left\{A_{i}\right\}_{1 \leq i \leq n} \subset \mathscr{B}(E)$. We call $\bar{Q}$ the stationary extension of $Q$ to $\overline{\mathbf{C}}_{E}$. For any $s \leq t$, let $\mathscr{F}_{t}^{s}:=\sigma\left(\overline{\mathbf{C}}_{E} \ni w \mapsto w(u): s \leq u \leq t\right)$. For a probability measure $\bar{Q}$ on $\overline{\mathbf{C}}_{E}$, let $\bar{Q}_{w-}$ be the regular conditional distribution of $\bar{Q}$ given $\mathscr{F}_{0}^{-\infty}$. Moreover, let Ent $_{\mathscr{F}_{1}^{0}}$ be the Kullback-Leibler divergence (i.e. relative entropy) on the $\sigma$-field $\mathscr{F}_{1}^{0}$; that is, for any two probability measures $\mu_{1}, \mu_{2}$ on $\mathbf{C}_{E}$,

$$
\operatorname{Ent}_{\mathscr{F}_{1}^{0}}\left(\mu_{1} \mid \mu_{2}\right):= \begin{cases}\int_{\mathbf{C}_{E}}(h \log h) \mathrm{d} \mu_{2}, & \text { if }\left.\mathrm{d} \mu_{1}\right|_{\mathscr{F}_{1}^{0}}=\left.h \mathrm{~d} \mu_{2}\right|_{\mathscr{F}_{1}^{0}}, \\ \infty, & \text { otherwise. }\end{cases}
$$

Now, for a standard Markov process on $E$ with $\left\{P^{x}: x \in E\right\} \subset \mathscr{P}\left(\mathbf{C}_{E}\right)$, where $P^{x}$ stands for the distribution of the process starting at $x$, the process level entropy function of DonskerVaradhan is given by

$$
H(Q):= \begin{cases}\int_{\overline{\mathbf{C}}_{E}} \operatorname{Ent}_{\mathscr{F}_{1}^{0}}\left(\bar{Q}_{w-} \mid P^{w(0)}\right) \bar{Q}(\mathrm{~d} w), & \text { if } Q \in \mathscr{P}^{s}\left(\mathbf{C}_{E}\right), \\ \infty, & \text { otherwise. }\end{cases}
$$

Then the Donsker-Varadhan level 2 entropy function is defined as

$$
J(\nu):=\inf \left\{H(Q): Q \in \mathscr{P}^{s}\left(\mathbf{C}_{E}\right), Q(w(0) \in \cdot)=\nu\right\}, \quad \nu \in \mathscr{P}(E) .
$$

This function has compact level sets in $\mathscr{P}(E)$ under the $\tau$ - (hence the weak) topology, see for instance [20, 21]. For any $\nu \in \mathscr{P}(E)$, let $\left(X_{t}^{\nu}\right)_{t \geq 0}$ be the Markov process with initial distribution $\nu$. Consider its empirical measure

$$
L_{t}^{\nu}:=\frac{1}{t} \int_{0}^{t} \delta_{X_{s}^{\nu}} \mathrm{d} s, \quad t>0 .
$$

When $\nu=\delta_{x}$, we denote $X_{t}^{\nu}=X_{t}^{x}$ and $L_{t}^{\nu}=L_{t}^{x}$. Let $\mu$ be an invariant probability measure of $P_{t}$, where $P_{t}$ is the Markov semigroup given by

$$
P_{t} f(x)=\mathbb{E}\left[f\left(X_{t}^{x}\right)\right], \quad x \in E, t \geq 0, f \in \mathscr{B}_{b}(E) .
$$

We write $f \in \mathscr{D}_{\mu}(\mathscr{A})$ if $f \in L^{\infty}(\mu)$ and there exists $g \in L^{\infty}(\mu)$ such that $P_{t} f-f=\int_{0}^{t} P_{s} g \mathrm{~d} s$ holds $\mu$-a.e. for all $t \geq 0$. In this case, we denote $\mathscr{A} f=g$. We have the following formula for $J$. 
T0 Theorem 3.2 ([21], Proposition B.10 and Corollary B.11). Assume that $P_{t}$ has a unique invariant probability measure $\mu$. Then

$$
J(\nu)= \begin{cases}\sup \left\{\int_{E} \frac{-\mathscr{A f}}{f} \mathrm{~d} \nu: 1 \leq f \in \mathscr{D}_{\mu}(\mathscr{A})\right\}, & \text { if } \nu \ll \mu, \\ \infty, & \text { otherwise }\end{cases}
$$

In particular, if the Markov process is associated with a symmetric Dirichlet form $(\mathscr{E}, \mathscr{D}(\mathscr{E}))$ in $L^{2}(\mu)$, then

$$
J(\nu)= \begin{cases}\mathscr{E}\left(h^{\frac{1}{2}}, h^{\frac{1}{2}}\right), & \text { if } \nu=h \mu, h^{\frac{1}{2}} \in \mathscr{D}(\mathscr{E}), \\ \infty, & \text { otherwise. }\end{cases}
$$

We now recall another result due to [21] on the LDP for uniformly integrable Markov semigroups, which will be used in the proof of Theorem 2.3. Let $p \geq 1$ and let $P$ be a bounded linear operator on $L^{p}(\mu)$. We call $P$ uniformly integrable in $L^{p}(\mu)$ if

$$
\lim _{R \rightarrow \infty} \sup _{\mu\left(|f|^{p}\right) \leq 1} \mu\left(|P f|^{p} 1_{\{|P f|>R\}}\right)=0 .
$$

This LDP is established under the $\tau$-topology induced by $f \in \mathscr{B}_{b}(E)$, and hence also holds under the weak topology. Let $\nu \in I_{q, L}:=\left\{\nu=h \mu:\|h\|_{L^{q}(\mu)} \leq L\right\}$ for $q, L \in(1, \infty)$.

T2 Theorem 3.3 ([21], Theorem 5.1). Assume that the Markov semigroup $P_{t}$ has a unique invariant probability measure $\mu$, and there exists $T \in(1, \infty)$ and $p \in(1, \infty)$ such that $P_{T}$ is $\mu$ irreducible and uniformly integrable in $L^{p}(\mu)$. Then $\left\{L_{t}^{\nu}\right\}_{\nu \in I_{q, L}} \in L D P(J)$ under the $\tau$-topology for all $q, L \in(1, \infty)$.

The next result due to [20] provides criteria on the LDP using the hitting time to compact sets, which will be used in the proofs of Theorem 2.1 and Theorem 2.2. For any set $K \subset E$ and any $x \in E$, let

$$
\tau_{K}^{x}:=\inf \left\{t \geq 0: X^{x}(t) \in K\right\},
$$

where $X^{x}(t)$ is the Markov process starting at $x$. We will use the following conditions:

(D1) For any $\lambda>0$ there exists a compact set $K \subset E$ such that

$$
\sup _{x \in E} \mathbb{E}\left[\mathrm{e}^{\lambda \tau_{K}^{x}}\right]<\infty .
$$

(D2) For any $\lambda>0$ there exist a constant $s>0$ and a compact set $K \subset E$ such that for any compact set $K^{\prime} \subset E$,

Wu2

$$
\sup _{x \in K} \mathbb{E}\left[\mathrm{e}^{\lambda \tau_{K}^{X^{x}(s)}}\right]<\infty, \sup _{x \in K^{\prime}} \mathbb{E}\left[\mathrm{e}^{\lambda \tau_{K}^{x}}\right]<\infty .
$$

T3 Theorem 3.4 ([20], Theorems 1.1,1.2). Assume that $P_{t}$ is a Feller Markov semigroup. 
(1) (D1) implies $\left\{L_{t}^{\nu}\right\}_{\nu \in \mathscr{P}(E)} \in L D P_{u}(J)$, and the inverse holds when $E$ is locally compact. If moreover $P_{t}$ is strong Feller and $\mu$-irreducible for some $t>0$, then $\left\{L_{t}^{\nu}\right\}_{\nu \in \mathscr{P}(E)} \in L D P(J)$ if and only if (D1) holds.

(2) (D2) implies $\left\{L_{t}^{x}\right\}_{x \in D} \in L D P_{u}(J)$ for any compact set $D \subset E$, and the inverse holds provided $E$ is locally compact. If $P_{t}$ is strong Feller and $\mu$-irreducible for some $t>0$, then $\left\{L_{t}^{x}\right\}_{x \in D} \in L D P(J)$ for compact $D \subset E$ if and only if (D2) holds.

Finally, we introduce the following approximation lemma which is easy to prove but useful in applications, see for instance [4, Theorems 4.2.16, 4.2.23], and see also [14, Theorem 3.2] for a stronger version called generalized contraction principle.

L1.1 Lemma 3.5 (Approximation Lemma for LDP). Let $\left\{\left(L_{t}^{\nu}\right)_{t>0},\left(\bar{L}_{t}^{\nu}\right)_{t>0}: \nu \in \mathscr{I}\right\}$ be two families of stochastic processes on a Polish space $(E, \rho)$ for an index set $\mathscr{I}$. If $\left(\bar{L}_{t}^{\nu}\right)_{\nu \in \text { scrI }} \in$ $L D P_{u}(J)$ (respectively $\left.L D P_{l}(J)\right)$ and

$$
\lim _{t \rightarrow \infty} \frac{1}{\lambda(t)} \sup _{\nu \in \mathscr{A}} \log \mathbb{P}\left(\rho\left(L_{t}^{\nu}, \bar{L}_{t}^{\nu}\right)>\delta\right)=-\infty, \quad \delta>0
$$

then $\left(L_{t}^{\nu}\right)_{\nu \in \mathscr{I}} \in \operatorname{LDP}_{u}(J)\left(\right.$ respectively $\left.L D P_{l}(J)\right)$.

\section{Proofs of main results}

To establish the LDP for $L_{t}^{\nu}$, we will compare (3.1) with a reference equation:

$\mathrm{BE}$

$$
\mathrm{d} \bar{X}^{\nu}(t)=\left\{A \bar{X}^{\nu}(t)+\bar{b}\left(\bar{X}_{t}^{\nu}\right)\right\} \mathrm{d} t+\bar{\sigma}\left(\bar{X}_{t}^{\nu}\right) \mathrm{d} W(t), \quad \bar{X}_{0}^{\nu}=X_{0}^{\nu},
$$

where $\bar{b}: \mathscr{C} \rightarrow \mathbb{H}, \quad \bar{\sigma}: \mathscr{C} \rightarrow \mathbb{L}(\mathbb{H})$ are measurable such that this equation has a unique mild segment solution for any initial value in $\mathscr{C}$, which is thus a Markov process on $\mathscr{C}$. In applications, the coefficients in (4.1) will be given by the limit of $b_{t}\left(\cdot, \nu_{t}\right)$ and $\sigma_{t}\left(\cdot, \nu_{t}\right)$ as $t \rightarrow \infty$, where $b_{t}$ and $\sigma_{t}$ are in (3.1) and $\nu_{t}:=\mathscr{L}_{X_{t}^{\nu}}$. Now, let

$$
\bar{L}_{t}^{\nu}=\frac{1}{t} \int_{0}^{t} \delta_{\bar{X}_{s}^{\nu}} \mathrm{d} s, \quad t>0 .
$$

We have the following result.

TM Theorem 4.1. Assume that (3.1) and (4.1) are well-posed for any initial value $X_{0}$ with $\mathscr{L}_{X_{0}} \in$ $\mathscr{I}$ and $\mathscr{L}_{\bar{X}_{0}} \in \Psi(\mathscr{I})$ respectively, where $\mathscr{I}$ is a non-empty subset of $\mathscr{P}(\mathscr{C})$ and $\Psi: \mathscr{I} \rightarrow \mathscr{P}(\mathscr{C})$ is a map. If $\left\{\bar{L}_{t}^{\nu}\right\}_{\nu \in \Psi(\mathscr{I})} \in L D P_{u}(J)$ (respectively $\left.L D P_{l}(J)\right)$ under the weak topology, and

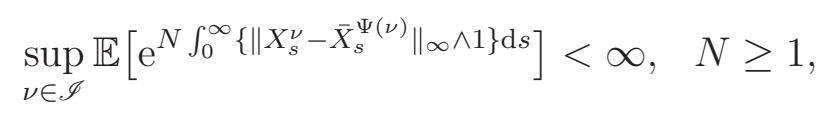

then $\left\{L_{t}^{\nu}\right\}_{\nu \in \mathscr{I}} \in L D P_{u}(J)$ (respectively $\left.L D P_{l}(J)\right)$ under the weak topology. 
Proof. Consider the probability distance

$$
\rho\left(\Lambda_{1}, \Lambda_{2}\right):=\inf _{\Pi \in \mathscr{C}\left(\Lambda_{1}, \Lambda_{2}\right)} \int_{\mathscr{C} \times \mathscr{C}}\left\{\|\xi-\eta\|_{\infty} \wedge 1\right\} \Pi(\mathrm{d} \xi, \mathrm{d} \eta)
$$

on $\mathscr{P}(\mathscr{C})$. It is well known that $\rho$ induces the weak topology on $\mathscr{P}(\mathscr{C})$. Since

$$
\rho\left(L_{t}^{\nu}, \bar{L}_{t}^{\bar{\nu}}\right) \leq \frac{1}{t} \int_{0}^{t}\left\{\left\|X_{s}^{\nu}-\bar{X}_{s}^{\Psi(\nu)}\right\|_{\infty} \wedge 1\right\} \mathrm{d} s, \quad t>0,
$$

(4.2) implies

$$
\begin{aligned}
& \lim _{t \rightarrow \infty} \frac{1}{t} \sup _{\nu \in \mathscr{I}} \log \mathbb{P}\left(\rho\left(L_{t}^{\nu}, \bar{L}_{t}^{\Psi(\nu)}\right)>\delta\right) \\
& \leq \lim _{t \rightarrow \infty} \frac{1}{t} \sup _{\nu \in \mathscr{I}} \log \mathbb{P}\left(N \int_{0}^{t}\left\{\left\|X_{s}^{\nu}-\bar{X}_{s}^{\Psi(\nu)}\right\|_{\infty} \wedge 1\right\} \mathrm{d} s>t N \delta\right) \\
& \leq-N \delta, \quad N \geq 1, \delta>0 .
\end{aligned}
$$

Therefore,

$$
\lim _{t \rightarrow \infty} \frac{1}{t} \sup _{\nu \in \mathscr{I}} \log \mathbb{P}\left(\rho\left(L_{t}^{\nu}, \bar{L}_{t}^{\Psi(\nu)}\right)>\delta\right)=-\infty, \quad \delta>0 .
$$

Then the desired assertion follows from Lemma 3.5 with $\bar{L}_{t}^{\Psi(\nu)}$ replacing $\bar{L}_{t}^{\nu}$.

\subsection{Proof of Theorem 2.1}

Obviously, condition $\left(H_{1}\right)$ implies that the SDE

$$
\mathrm{d} \bar{X}(t)=b(\bar{X}(t), \bar{\mu}) \mathrm{d} t+\sigma(\bar{\mu}) \mathrm{d} W(t)
$$

is well-posed and the solution is a Markov Feller process, where $\bar{\mu}$ is the unique invariant probability measure of $P_{t}^{*}$. Let $\bar{X}^{x}(t)$ denote the solution starting at $x$. According to Theorem 3.4 and Theorem 4.1, we only need to prove the following assertions:

(a) For any $\lambda>0$, there exist a constant $s>0$ and compact set $K \subset \mathbb{R}^{d}$, such that (3.14) holds for any compact set $K^{\prime} \subset \mathbb{R}^{d}$ and

$$
\tau_{K}^{x}:=\inf \left\{t \geq 0: \bar{X}^{x}(t) \in K\right\}, \quad x \in \mathbb{R}^{d} .
$$

(b) For any $N \geq 1$,

$$
\sup _{\nu \in \mathscr{B}_{r, R}} \mathbb{E} \mathrm{e}^{N \int_{0}^{\infty}\left\{1 \wedge\left|X^{\nu}(s)-\bar{X}^{0}(s)\right|^{2}\right\} \mathrm{d} s}<\infty
$$

(c) Under (2.4), for any $\lambda>0$ there exists a compact set $K \subset \mathbb{R}^{d}$ such that (3.13) holds for $\bar{X}$, and

$$
\sup _{\nu \in \mathscr{P}_{2}\left(\mathbb{R}^{d}\right)} \mathbb{E}^{N \int_{0}^{\infty}\left\{1 \wedge\left|X^{\nu}(s)-\bar{X}^{\nu}(s)\right|^{2}\right\} \mathrm{d} s}<\infty, \quad N \geq 1 .
$$


Indeed, by Theorem 3.4(2), (a) implies the upper LDP (LDP if $\bar{P}_{t}$ is strong Feller and $\bar{\mu}-$ irreducible) for $\bar{L}_{t}^{x}$ locally uniformly in $x$, in particular, $L_{t}^{0}$ satisfies the upper LDP (LDP if $\bar{P}_{t}$ is strong Feller and $\bar{\mu}$-irreducible). Combining this with (b) and Theorem 4.1 for $\mathscr{I}=\mathscr{B}_{r, R}$ and $\Psi(\nu):=\delta_{0}$, we prove the desired assertion for $L_{t}^{\nu}$ with $\nu \in \mathscr{B}_{r, R}$. Finally, by Theorem 3.4(1) and Theorem 4.1 with $\mathscr{I}=\mathscr{P}_{2}\left(\mathbb{R}^{d}\right)$ and $\Psi(\nu)=\nu,(\mathrm{c})$ implies the upper LDP (LDP if $\bar{P}_{t}$ is strong Feller and $\bar{\mu}$-irreducible) for $L_{t}^{\nu}$ uniformly in $\nu \in \mathscr{P}_{2}\left(\mathbb{R}^{d}\right)$.

Proof of (a). By $\left(H_{1}\right)$, there exist constants $\alpha, \beta>0$ such that

$$
\mathrm{d}|\bar{X}(t)|^{2} \leq 2\left\{\alpha-\beta|\bar{X}(t)|^{2}\right\} \mathrm{d} t+2\langle\bar{X}(t), \sigma(\bar{\mu}) \mathrm{d} W(t)\rangle .
$$

Let $\theta=\|\sigma\|_{\infty}^{2}$. Then for any $\varepsilon \in(0, \beta / \theta)$, there exist constants $c_{1}, c_{2}>0$ such that

$$
\begin{aligned}
\mathrm{de}^{\varepsilon|\bar{X}(t)|^{2}} & \leq 2 \varepsilon\left\{\alpha-(\beta-\varepsilon \theta)|\bar{X}(t)|^{2}\right\} \mathrm{e}^{\varepsilon|\bar{X}(t)|^{2}} \mathrm{~d} t+\mathrm{d} M(t) \\
& \leq\left\{c_{1}-c_{2} \mathrm{e}^{\varepsilon|\bar{X}(t)|^{2}}\right\} \mathrm{d} t+\mathrm{d} M(t)
\end{aligned}
$$

for some martingale $M(t)$. So,

$$
\mathbb{E} \mathrm{e}^{\varepsilon\left|\bar{X}^{x}(t)\right|^{2}} \leq \mathrm{e}^{\varepsilon|x|^{2}}+\frac{c_{1}}{c_{2}}, \quad x \in \mathbb{R}^{d} .
$$

To estimate $\tau_{K}^{x}$ for $K:=B_{0}(N)$, we take $N \geq N_{0}:=(2 \alpha / \beta)^{\frac{1}{2}}$. Then (4.4) implies

$$
\mathrm{d}|\bar{X}(t)|^{2} \leq-\beta|\bar{X}(t)|^{2} \mathrm{~d} t+2\left\langle\bar{X}^{x}(t), \sigma(\bar{\mu}) \mathrm{d} W(t)\right\rangle, \quad t \leq \tau_{K}^{x} .
$$

For any $\delta>0$, we obtain

$$
\begin{aligned}
& \mathbb{E} \mathrm{e}^{\delta \int_{0}^{t \wedge \tau_{K}^{x}}\left|\bar{X}^{x}(s)\right|^{2} \mathrm{~d} s} \leq \mathrm{e}^{\delta \beta^{-1}|x|^{2}} \mathbb{E} \mathrm{e}^{2 \delta \beta^{-1} \int_{0}^{t \wedge \tau_{K}^{x}}\left\langle\bar{X}^{x}(s), \sigma\left(\bar{X}^{x}(s), \mu\right) \mathrm{d} W(s)\right\rangle} \\
& \leq \mathrm{e}^{\delta \beta^{-1}|x|^{2}}\left(\mathbb{E} \mathrm{e}^{8 \delta^{2} \beta^{-2} \theta \int_{0}^{t \wedge \tau_{K}^{x}}\left|\bar{X}^{x}(s)\right|^{2} \mathrm{~d} s}\right)^{\frac{1}{2}} .
\end{aligned}
$$

Thus, taking $\delta \leq \frac{\beta^{2}}{8 \theta}$ we arrive at

$$
\mathbb{E} \mathrm{e}^{\delta N^{2}\left(t \wedge \tau_{K}^{x}\right)} \leq \mathbb{E} \mathrm{e}^{\delta \int_{0}^{t \wedge \tau_{K}^{x}}\left|\bar{X}^{x}(s)\right|^{2} \mathrm{~d} s} \leq \mathrm{e}^{2 \delta \beta^{-1}|x|^{2}}
$$

Letting $t \uparrow \infty$ implies

$$
\mathbb{E} \mathrm{e}^{\delta N^{2} \tau_{K}^{x}} \leq \mathrm{e}^{2 \delta \beta^{-1}|x|^{2}}, \quad x \in \mathbb{R}^{d}, N \geq N_{0} .
$$

Combining this with the Markov property and (4.5), when $\delta \leq \frac{\varepsilon \beta}{2}$ we have

$$
\mathbb{E} \mathrm{e}^{\delta N^{2} \tau_{K}^{\bar{X}^{x}(s)}} \leq \mathbb{E}^{2 \delta \beta^{-1}\left|\bar{X}^{x}(s)\right|^{2}} \leq \mathrm{e}^{\varepsilon|x|^{2}}+\frac{c_{1}}{c_{2}}, \quad x \in \mathbb{R}^{d}, s \geq 0, N \geq N_{0} .
$$

Therefore, for any $\lambda>0$ there exists compact $K \subset \mathbb{R}^{d}$ such that (3.13) holds. 
Proof of (b). Simply denote $X(t)=X^{\nu}(t), \bar{X}(t)=\bar{X}^{0}(t)$ and $\nu_{t}=\mathscr{L}_{X^{\nu}(t)}=P_{t}^{*} \nu$ for $\nu \in \mathscr{B}_{r, R}$. By $\left(H_{1}\right),(2.2)$ and Itô's formula, we obtain

$$
\begin{aligned}
\mathrm{d}|X(t)-\bar{X}(t)|^{2} \leq & \left\{-\kappa_{1}|X(t)-\bar{X}(t)|^{2}+\kappa_{2} \mathrm{e}^{-\left(\kappa_{1}-\kappa_{2}\right) t} \mathbb{W}_{2}(\bar{\mu}, \nu)^{2}\right\} \mathrm{d} t \\
& +2\left\langle X(t)-\bar{X}(t),\left\{\sigma\left(\nu_{t}\right)-\sigma(\bar{\mu})\right\} \mathrm{d} W(t)\right\rangle .
\end{aligned}
$$

Letting $\gamma(t)=\frac{|X(t)-\bar{X}(t)|^{2}}{1+|X(t)-X(t)|^{2}}$, we derive

$$
\begin{aligned}
& \mathrm{d} \log \left(1+|X(t)-\bar{X}(t)|^{2}\right) \leq\left\{-\kappa_{1} \gamma(t)+\kappa_{2} \mathrm{e}^{-\left(\kappa_{1}-\kappa_{2}\right) t} \mathbb{W}_{2}(\bar{\mu}, \nu)^{2}\right\} \mathrm{d} t \\
& +\frac{2}{1+|X(t)-\bar{X}(t)|^{2}}\left\langle X(t)-\bar{X}(t),\left\{\sigma\left(\nu_{t}\right)-\sigma(\bar{\mu})\right\} \mathrm{d} W(t)\right\rangle .
\end{aligned}
$$

We deduce from this and (2.2) that for any $\lambda>0$,

$$
\begin{aligned}
& \mathrm{e}^{-\frac{\lambda \kappa_{2}}{\kappa_{1}-\kappa_{2}} \mathbb{W}_{2}(\bar{\mu}, \nu)^{2}} \mathbb{E}\left[\mathrm{e}^{\lambda \kappa_{1} \int_{0}^{t} \gamma(s) \mathrm{d} s}\right] \\
& \leq \mathbb{E}\left[\left(1+\left|X_{0}\right|^{2}\right)^{\lambda} \mathrm{e}^{\lambda \int_{0}^{t} \frac{2}{1+|X(s)-\bar{X}(s)|^{2}}\left\langle X(s)-\bar{X}(s),\left\{\sigma\left(\nu_{s}\right)-\sigma(\mu)\right\} \mathrm{d} W(s)\right\rangle}\right] \\
& \leq \mathbb{E}\left[\left(1+\left|X_{0}\right|^{2}\right)^{\lambda}\left(\mathbb{E}\left[\mathrm{e}^{8 \kappa_{2} \lambda^{2} \int_{0}^{t} \gamma(s) \mathbb{W}_{2}\left(\nu_{s}, \bar{\mu}\right)^{2} \mathrm{~d} s}\right] \mid \mathscr{F}_{0}\right)^{\frac{1}{2}}\right] \\
& \leq\left\{\nu\left(\left(1+|\cdot|^{2}\right)^{2 \lambda}\right)\right\}^{\frac{1}{2}}\left(\mathbb{E}\left[\mathrm{e}^{8 \kappa_{2} \lambda^{2} \mathbb{W}_{2}(\nu, \bar{\mu})^{2} \int_{0}^{t} \gamma(s) \mathrm{e}^{-\left(\kappa_{1}-\kappa_{2}\right) s} \mathrm{~d} s}\right]\right)^{\frac{1}{2}} \\
& \leq C(\lambda, R)\left(\mathbb{E}\left[\mathrm{e}^{\lambda \kappa_{1} \int_{0}^{t} \gamma(s) \mathrm{d} s}\right]\right)^{\frac{1}{2}}, \quad t>0
\end{aligned}
$$

holds for some constant $C(\lambda, R)>0$, where the last step is due to $\gamma(s) \leq 1$ and $\nu \in \mathscr{B}_{r, R}$. Therefore,

$$
\sup _{\nu \in \mathscr{B}_{r, R}} \mathbb{E}\left[\mathrm{e}^{\lambda \kappa_{1} \int_{0}^{\infty} \frac{\left|X^{\nu}(s)-\bar{X}^{0}(s)\right|^{2}}{1+\left|X^{\nu}(s)-\bar{X}^{0}(s)\right|^{2}} \mathrm{~d} s}\right]<\infty, \quad \lambda>0,
$$

which implies (b).

Proof of (c). Let (2.4) hold. Then there exist constants $c_{1}, c_{2}>0$ such that

$$
\mathrm{de}^{|\bar{X}(t)|^{2}} \leq\left\{c_{1}-c_{2}|\bar{X}(t)|^{2+\varepsilon} \mathrm{e}^{|\bar{X}(t)|^{2}}\right\} \mathrm{d} t+2 \mathrm{e}^{|\bar{X}(t)|^{2}}\langle\bar{X}(t), \sigma(\bar{\mu}) \mathrm{d} W(t)\rangle .
$$

This implies

$$
h_{x}(t):=\mathbb{E} \mathrm{e}^{\left|\bar{X}^{x}(t)\right|^{2}} \leq c_{1} t+\mathrm{e}^{|x|^{2}}<\infty, \quad t \geq 0, x \in \mathbb{R}^{d} .
$$

Moreover, by Jensen's inequality and the convexity of $[1, \infty) \ni r \mapsto r \log ^{1+\varepsilon / 2} r$, we deduce from (4.8) that

$$
h_{x}(t) \leq h_{x}(0)+c_{1} t-c_{2} \int_{0}^{t} h_{x}(s) \log ^{1+\varepsilon / 2} h_{x}(s) \mathrm{d} s, \quad t \geq 0
$$

This and the comparison theorem imply $h_{x}(t) \leq \psi_{x}(t)$, where $\psi_{x}(t)$ solves the ODE

$$
\psi^{\prime}(t)=c_{1}-c_{2} \psi(t) \log ^{1+\varepsilon / 2} \psi(t), \quad \psi(0)=h_{x}(0)=\mathrm{e}^{|x|^{2}} .
$$


So,

$$
\sup _{x \in \mathbb{R}^{d}} h_{x}(t) \leq \sup _{\psi(0) \geq 1} \psi(t)=: c(t)<\infty .
$$

On the other hand, by (4.8), there exist constants $N_{0}, \beta>0$ such that for any $N \geq N_{0}$ and $K=B_{0}(N)$, we have

$$
\mathrm{de}^{\left|\bar{X}^{x}(t)\right|^{2}} \leq-\beta\left|\bar{X}^{x}(t)\right|^{2+\varepsilon} \mathrm{e}^{\left|\bar{X}^{x}(t)\right|^{2}} \mathrm{~d} t+2 \mathrm{e}^{\left|\bar{X}^{x}(t)\right|^{2}}\left\langle\bar{X}^{x}(t), \sigma(\bar{\mu}) \mathrm{d} W(t)\right\rangle, \quad t \leq \tau_{K}^{x} .
$$

Combining this with (4.6) and using the Markov property, when $2 \delta \leq \beta^{2}$ we arrive at

$$
\begin{aligned}
& \mathbb{E}\left[\mathrm{e}^{\delta N^{2} \tau_{K}^{x}}\right] \leq \mathrm{e}^{\delta N^{2}}+\mathbb{E}\left[\mathrm{e}^{\delta N^{2} \tau_{K}^{x}} 1_{\left\{\tau_{K}^{x} \geq 1\right\}}\right] \\
& \leq \mathrm{e}^{\delta N^{2}}+\mathbb{E}\left[\mathrm{e}^{\delta N^{2}\left(1+\tau_{K}^{\bar{X}^{x}(1)}\right)} 1_{\left\{\tau_{K}^{x} \geq 1\right\}}\right] \\
& \leq \mathrm{e}^{\delta N^{2}}\left(1+\mathbb{E} \mathrm{e}^{\left|\bar{X}^{x}(1)\right|^{2}}\right) \leq \mathrm{e}^{\delta N^{2}}(1+c(1))<\infty, \quad x \in \mathbb{R}^{d}, N \geq N_{0} .
\end{aligned}
$$

Therefore, for any $\lambda>0$, there exists compact set $K$ such that (3.13) holds.

Finally, repeating the proof of (4.9) using $X^{\nu}(t)$ replacing $\bar{X}^{x}(t)$, we derive

$$
\sup _{\nu \in \mathscr{P}_{2}\left(\mathbb{R}^{d}\right)} \mathbb{E}\left[\mathrm{e}^{\left|X^{\nu}(1)\right|^{2}}\right]<\infty .
$$

This together with (4.9) yields

$$
\sup _{\nu \in \mathscr{P}_{2}\left(\mathbb{R}^{d}\right)} \mathbb{E}\left[\mathrm{e}^{\left|X^{\nu}(1)\right|^{2}}+\mathrm{e}^{\left|\bar{X}^{\nu}(1)\right|^{2}}\right]<\infty
$$

On the other hand, as in (4.7) but integrating from time 1, we obtain

$$
\begin{aligned}
& \mathrm{e}^{-\frac{\lambda \kappa_{2}}{\kappa_{1}-\kappa_{2}} \mathbb{W}_{2}(\bar{\mu}, \nu)^{2}} \mathbb{E}\left[\mathrm{e}^{\lambda \kappa_{1} \int_{1}^{t} \frac{\left|X^{\nu}(s)-\bar{X}^{\nu}(s)\right|^{2}}{1+\left|X^{\nu}(s)-\bar{X}^{\nu}(s)\right|^{2}} \mathrm{~d} s}\right] \\
& \leq \mathbb{E}\left[\left(1+\left|X^{\nu}(1)-\bar{X}^{\nu}(1)\right|^{2}\right)^{\lambda} \mathrm{e}^{\lambda \int_{1}^{t} \frac{2}{1+\left|X^{\nu}(s)-\bar{X}^{\nu}(s)\right|^{2}}\left\langle X^{\nu}(s)-\bar{X}^{\nu}(s),\left\{\sigma\left(\nu_{s}\right)-\sigma(\bar{\mu})\right\} \mathrm{d} W(s)\right\rangle}\right] \\
& \leq\left\{\mathbb{E}\left[\left(1+\left|X^{\nu}(1)-\bar{X}^{\nu}(1)\right|^{2}\right)^{2 \lambda}\right]\right\}^{\frac{1}{2}}\left(\mathbb{E}\left[\mathrm{e}^{\lambda \kappa_{1} \mathbb{W}_{2}\left(P_{1}^{*} \nu, \bar{\mu}\right)^{2} \int_{1}^{t} \frac{\left|X^{\nu}(s)-\bar{X}^{\nu}(s)\right|^{2} \mathrm{e}^{-\left(\kappa_{1}-\kappa_{2}\right) s}}{1+\left|X^{\nu}(s)-\bar{X}^{\nu}(s)\right|^{2}} \mathrm{~d} s}\right]\right)^{\frac{1}{2}}, \quad t>1 .
\end{aligned}
$$

Combining this with (4.11), we derive

$$
\sup _{\nu \in \mathscr{P}_{2}\left(\mathbb{R}^{d}\right)} \mathbb{E} \mathrm{e}^{\lambda \kappa_{1} \int_{1}^{\infty} \frac{\left|X^{\nu}(s)-\bar{X}^{\nu}(s)\right|^{2}}{1+\left|X^{\nu}(s)-\bar{X}^{\nu}(s)\right|^{2}} \mathrm{~d} s}<\infty, \quad \lambda \geq 1 .
$$

Therefore, (c) holds.

\subsection{Proof of Theorem 2.2}

As explained in the beginning of Subsection 4.1 that we only need to verify (a) and (b) in the last subsection for the present model. Comparing with the finite-dimensional case, the main difficulty is that bounded sets are no longer compact. To construct compact sets, let $\left\{e_{i}\right\}_{i \geq 1}$ 
be the eigenbasis of $A$; i.e. it is an orthonromal basis of $\mathbb{H}$ such that $A e_{i}=-\lambda_{i} e_{i}, i \geq 1$. For any $N>0$, the set

$$
K:=B_{0, \gamma}(N)=\left\{x \in \mathbb{H}:|x|_{\gamma}^{2}:=\sum_{i=1}^{\infty}\left\langle x, e_{i}\right\rangle^{2} \lambda_{i}^{\gamma} \leq N^{2}\right\}
$$

is a compact set in $\mathbb{H}$.

Proof of (a). Simply denote $\bar{X}(t)=\bar{X}^{x}(t)$ and $\tau_{K}=\tau_{K}^{x}:=\inf \left\{t \geq 0: \bar{X}^{x}(t) \in K\right\}$. By $\left(H_{2}\right)$ and (2.8), we may apply Itô's formula to

$$
\psi(\bar{X}(t)):=\left\langle(-A)^{\gamma-1} \bar{X}(t), \bar{X}(t)\right\rangle=\sum_{i=1}^{\infty}\left\langle\bar{X}(t), e_{i}\right\rangle^{2} \lambda_{i}^{\gamma-1}
$$

such that for some constants $d_{1}, d_{2}>0$

$$
\mathrm{d} \psi(\bar{X}(t)) \leq\left(d_{1}-d_{2}|\bar{X}(t)|_{\gamma}^{2}\right) \mathrm{d} t+\mathrm{d} M(t)
$$

where $M(t):=2 \sum_{i=1}^{\infty} \lambda_{i}^{\gamma-1}\left\langle\bar{X}(t), e_{i}\right\rangle\left\langle\sigma(\bar{\mu}) \mathrm{d} W(t), e_{i}\right\rangle$ for an orthonormal basis $\left\{e_{i}\right\}_{i \geq 1}$ of $\mathbb{H}$. Let $N \geq N_{0}:=\left(2 d_{1} / d_{2}\right)^{\frac{1}{2}}$, and consider $\tau_{K}$ for $K=B_{0, \gamma}(N)$. Then

$$
d_{1}-d_{2}|\bar{X}(t)|_{\gamma}^{2} \leq-d_{1}|\bar{X}(t)|_{\gamma}^{2}, \quad t \leq \tau_{K}
$$

Since $\sigma$ is bounded, by $\left(H_{2}\right)$ there exists a constant $c>0$ such that

$$
\langle M\rangle(t) \leq c \int_{0}^{t}|\bar{X}(s)|^{2} \mathrm{~d} s, \quad t \geq 0
$$

So, letting $\tau_{n}:=\inf \{t \geq 0:|\bar{X}(t)| \geq n\}$, we deduce form (4.12) and (4.13) that

$$
\begin{aligned}
& \mathbb{E} \mathrm{e}^{\int_{0}^{t \wedge \tau_{n} \wedge \tau_{K}} \delta d_{1}|X(s)|_{\gamma}^{2} \mathrm{~d} s} \leq \mathrm{e}^{\delta \psi(x)}\left(\mathbb{E}^{2 \delta^{2}\langle M\rangle\left(t \wedge \tau_{n} \wedge \tau_{K}\right)}\right)^{\frac{1}{2}} \\
& \leq \mathrm{e}^{\delta \psi(x)}\left(\mathbb{E} \mathrm{e}^{2 c \delta^{2} \int_{0}^{t \wedge \tau_{n} \wedge \tau_{K}}|\bar{X}(s)|_{\gamma}^{2} \mathrm{~d} s}\right)^{\frac{1}{2}}<\infty, \quad n \geq 1 .
\end{aligned}
$$

Taking $\delta \leq(2 c)^{-1}$ leads to

$$
\mathbb{E} \mathrm{e}^{\delta d_{1} N^{2}\left(t \wedge \tau_{n} \wedge \tau_{K}\right)} \leq \mathbb{E}^{\int_{0}^{t \wedge \tau_{n} \wedge \tau_{K}} \delta d_{1}|\bar{X}(s)|_{\gamma}^{2} \mathrm{~d} s} \leq \mathrm{e}^{2 \delta \psi(x)}, \quad t \geq 0, n \geq 1 .
$$

Letting $t, n \rightarrow \infty$ we derive

$$
\mathbb{E} \mathrm{e}^{\delta N^{2} d_{1} \tau_{K}} \leq \mathrm{e}^{2 \delta \psi(x)}, \quad x \in \mathbb{H} .
$$

Combining this with the Markov property, we obtain

$$
\mathbb{E} \mathrm{e}^{\delta N^{2} d_{1} \tau_{K}^{\bar{X}(s)}} \leq \mathbb{E} \mathrm{e}^{2 \delta \psi(\bar{X}(s))}
$$

and it is easy to see from (4.12) that the upper bound is locally bounded in $x$ when $\delta$ is small enough. Therefore, condition (a) is satisfied, since $N \geq N_{0}$ is arbitrary. 
Proof of (b). By $\left(H_{2}\right),(2.7)$, and Itô's formula, we have

$$
\begin{aligned}
\mathrm{d}\left|X^{\nu}(t)-\bar{X}^{0}(t)\right|^{2} \leq\{ & \left.-2\left(\lambda_{1}-\alpha_{1}\right)\left|X^{\nu}(t)-\bar{X}^{0}(t)\right|^{2}+2 \alpha_{2} \mathbb{W}_{2}\left(P_{t}^{*} \nu, \bar{\mu}\right)^{2}\right\} \mathrm{d} t \\
& +2\left\langle X^{\nu}(t)-\bar{X}^{0}(t),\left\{\sigma\left(P_{t}^{*} \nu\right)-\sigma(\bar{\mu})\right\} \mathrm{d} W(t)\right\rangle .
\end{aligned}
$$

The remainder of the proof is completely similar to that of (b) in the last subsection.

\subsection{Proof of Theorem 2.3}

Let $\theta \in\left[0, \lambda_{1}\right]$ such that $\kappa_{p}=\theta-\left(\alpha_{1}+\alpha_{2}\right) \mathrm{e}^{p \theta r_{0}}$.

\subsubsection{Proof of Theorem 2.3(1)}

For any $\nu_{1}, \nu_{2} \in \mathscr{P}_{p}(\mathscr{C})$, take $X_{0}^{\nu_{i}} \in L^{p}\left(\Omega \rightarrow \mathscr{C}, \mathscr{F}_{0}, \mathbb{P}\right)$ such that $\mathscr{L}_{X_{0}^{\nu_{i}}}=\nu_{i}, i=1,2$, and

$$
\mathbb{E}\left[\left\|X_{0}^{\nu_{1}}-X_{0}^{\nu_{2}}\right\|_{\infty}^{p}\right]=\mathbb{W}_{p}\left(\nu_{1}, \nu_{2}\right)^{p}
$$

Since $\sigma$ is constant, we have

$$
\mathrm{d}\left(X^{\nu_{1}}(t)-X^{\nu_{2}}(t)\right)=\left\{A\left(X^{\nu_{1}}(t)-X^{\nu_{2}}(t)\right)+b\left(X_{t}^{\nu_{1}}, P_{t}^{*} \nu_{1}\right)-b\left(X_{t}^{\nu_{2}}, P_{t}^{*} \nu_{2}\right)\right\} \mathrm{d} t, \quad t \geq 0 .
$$

By $\left(H_{3}\right)$ and noting that $\theta \in\left[0, \lambda_{1}\right]$, we obtain

$$
\begin{aligned}
& \mathrm{d}\left\{\left|X^{\nu_{1}}(t)-X^{\nu_{2}}(t)\right|^{p} \mathrm{e}^{p \theta t}\right\} \\
& =p \mathrm{e}^{p \theta t}\left|X^{\nu_{1}}(t)-X^{\nu_{2}}(t)\right|^{p-2}\left\{\left\langle X^{\nu_{1}}(t)-X^{\nu_{2}}(t),(\theta+A)\left(X^{\mu}(t)-X^{\nu_{2}}(t)\right)\right\rangle\right. \\
& \left.\quad+\left\langle X^{\nu_{1}}(t)-X^{\nu_{2}}(t), b\left(X_{t}^{\nu_{1}}, P_{t}^{*} \nu_{1}\right)-b\left(X_{t}^{\nu_{2}}, P_{t}^{*} \nu_{2}\right)\right\rangle\right\} \mathrm{d} t \\
& \leq p\left|X^{\nu_{1}}(t)-X^{\nu_{2}}(t)\right|^{p-1} \mathrm{e}^{p \theta t}\left\{\alpha_{1}\left\|X_{t}^{\nu_{1}}-X_{t}^{\nu_{1}}\right\|_{\infty}+\alpha_{2} \mathbb{W}_{p}\left(P_{t}^{*} \nu_{1}, P_{t}^{*} \nu_{2}\right)\right\} \mathrm{d} t, \quad t \geq 0 .
\end{aligned}
$$

Letting $\psi(t)=\left\|X_{t}^{\nu_{1}}-X_{t}^{\nu_{2}}\right\|_{\infty}^{p} \mathrm{e}^{p \theta t}$, we derive

$$
\begin{aligned}
& \psi(t) \leq \mathrm{e}^{p \theta r_{0}} \sup _{s \in\left[\left(t-r_{0}\right), t\right]}\left|X^{\nu_{1}}(s)-X^{\nu_{2}}(s)\right|^{p} \mathrm{e}^{p \theta s} \\
& \leq \mathrm{e}^{p \theta r_{0}}\left\|X_{0}^{\nu_{1}}-X_{0}^{\nu_{2}}\right\|_{\infty}^{p}+p \mathrm{e}^{p \theta r_{0}} \int_{0}^{t}\left\{\alpha_{1} \psi(s)+\alpha_{2} \mathrm{e}^{\theta s} \mathbb{W}_{p}\left(P_{s}^{*} \nu_{1}, P_{s}^{*} \nu_{2}\right) \psi(s)^{\frac{p-1}{p}}\right\} \mathrm{d} s .
\end{aligned}
$$

Combining this with (4.14) and

$$
\mathbb{W}_{p}\left(P_{t}^{*} \nu_{1}, P_{t}^{*} \nu_{2}\right)^{p} \leq \mathbb{E}\left\|X_{t}^{\nu_{1}}-X_{t}^{\nu_{2}}\right\|_{\infty}^{p}, \quad t \geq 0,
$$

we arrive at

$$
\mathbb{E}[\psi(t)] \leq \mathrm{e}^{p \theta r_{0}} \mathbb{W}_{p}\left(\nu_{1}, \nu_{2}\right)^{p}+p \mathrm{e}^{p \theta r_{0}}\left(\alpha_{1}+\alpha_{2}\right) \int_{0}^{t} \mathbb{E}[\psi(s)] \mathrm{d} s, \quad t \geq 0 .
$$

By Theorem 3.1 we have $\mathbb{E}[\psi(t)]<\infty, t>0$. Then Gronwall's lemma yields

$$
\mathbb{E}[\psi(t)] \leq\left\{\mathbb{W}_{p}\left(\nu_{1}, \nu_{2}\right)\right\}^{p} \mathrm{e}^{p \theta r_{0}+p\left(\alpha_{1}+\alpha_{2}\right) \mathrm{e}^{p \theta r_{0} t}}, \quad t \geq 0 .
$$

Therefore,

$$
\mathbb{W}_{p}\left(P_{t}^{*} \nu_{1}, P_{t}^{*} \nu_{2}\right)^{p} \leq \mathrm{e}^{-p \theta t} \mathbb{E}[\psi(t)] \leq\left\{\mathbb{W}_{p}\left(\nu_{1}, \nu_{2}\right)\right\}^{p} \mathrm{e}^{p \theta r_{0}-p \kappa_{p} t}, \quad t \geq 0 .
$$

When $\kappa_{p}>0$, it is standard that (2.12) implies the existence and uniqueness of $P_{t}^{*}$-invariant probability measure $\mu$ such that (2.13) holds, see, for instance, [18, Proof of Theorem 3.1(2)]. 


\subsubsection{Proof of Theorem $2.3(2)$}

Let $\kappa_{p}>0$. To prove the LDP, let $\bar{P}_{t}$ be the Markov semigroup for the stationary equation (2.11) and consider the LDP for $\bar{L}_{t}^{\nu}$. Since $\lambda>0$ implies $\sup _{r \in\left[0, \lambda_{1}\right]}\left(r-\alpha_{1} \mathrm{e}^{r r_{0}}\right)>0$ and noting that $\left(H_{3}\right)$ implies

$$
|b(\xi, \mu)-b(\eta, \mu)| \leq \alpha_{1}\|\xi-\eta\|_{\infty},
$$

by [1, Theorem 1.2] and $\kappa_{1} \geq \kappa_{p}>0$, the Markov semigroup $\bar{P}_{t}$ is hypercontractive. Thus, by the semigroup property and the interpolation theorem, for any $q>1$ there exists $t_{q}>0$ such that $\bar{P}_{t_{q}}$ is uniformly integrable in $L^{q}(\mu)$. Moreover, according to [16, Theorem 4.2.4], assumption $\left(H_{3}\right)$ implies that for any $t>r_{0}$, there exists a constant $c>0$ such that the following Harnack inequality holds:

$$
\left.\left(\bar{P}_{t_{0}} f(\eta)\right)^{2} \leq\left(\bar{P}_{t_{0}} f^{2}(\xi)\right)\right) \mathrm{e}^{c\|\xi-\eta\|_{\infty}^{2}}, \quad \xi, \eta \in \mathscr{C}, f \in \mathscr{B}_{b}(\mathscr{C}) .
$$

Obviously, $\bar{\mu}$ is also $\bar{P}_{t}$-invariant, then for any $B \in \mathscr{B}(\mathscr{C})$ such that $\bar{\mu}(B)>0$, we have $\bar{\mu}\left(\bar{P}_{t_{0}} 1_{B}\right)=\mu(B)>0$, so that there exits $\eta \in \mathscr{C}$ such that $\bar{P}_{t_{0}} 1_{B}(\eta)>0$. Then (4.16) implies $\bar{P}_{t_{0}} 1_{B}(\xi)>0$ for all $\xi \in \mathscr{C}$, so that $\bar{\mu}\left(1_{A} \bar{P}_{t_{0}} 1_{B}\right)>0$ for $\bar{\mu}(A), \bar{\mu}(B)>0$, i.e. $\bar{P}_{t_{0}}$ is $\bar{\mu}$-irreducible. Therefore, by Theorem 3.3 .

$$
\bar{L}_{t}^{\nu} \in L D P(J) \text { uniformly in } \nu=h \bar{\mu} \in \mathscr{P}(\mathscr{C}) \text { with }\|h\|_{L^{q}(\bar{\mu})} \leq R, \quad R>0 .
$$

Combining this with Theorem 4.1, it remains to show that for any $\varepsilon, R>0$,

(I) $\left\{\bar{L}_{t}^{\nu}\right\}_{\nu \in \mathscr{I}_{\varepsilon, R}} \in \operatorname{LDP}(J)$;

(II) For any $\delta>0$,

$$
\lim _{t \rightarrow \infty} \frac{1}{t} \sup _{\nu \in \mathscr{I}_{\varepsilon, R}} \log \mathbb{P}\left(\frac{1}{t} \int_{0}^{t}\left\{1 \wedge\left\|X_{s}^{\nu}-\bar{X}_{s}^{\nu}\right\|_{\infty}\right\} \mathrm{d} s>\delta\right)=-\infty .
$$

For (I). Observing that for any $\xi, \eta \in \mathscr{C}$ we have

$$
\mathrm{d}\left(\bar{X}^{\xi}(t)-\bar{X}^{\eta}(t)\right)=\left\{A\left(\bar{X}^{\xi}(t)-\bar{X}^{\eta}(t)\right)+b\left(\bar{X}_{t}^{\xi}, \bar{\mu}\right)-b\left(\bar{X}_{t}^{\eta}, \bar{\mu}\right)\right\} \mathrm{d} t,
$$

by the same reason leading to (4.15) we obatin

$$
\left\|\bar{X}_{t}^{\xi}-\bar{X}_{t}^{\eta}\right\|_{\infty}^{p} \mathrm{e}^{p \theta t} \leq \mathrm{e}^{p \theta r_{0}}\|\xi-\eta\|_{\infty}^{p}+\alpha_{1} p \mathrm{e}^{p \theta r_{0}}\left\|\bar{X}_{s}^{\xi}-\bar{X}_{s}^{\eta}\right\|_{\infty}^{p} \mathrm{e}^{p \theta s} \mathrm{~d} s, \quad t \geq 0 .
$$

Noting that $\kappa_{p} \leq \theta-\alpha_{1} \mathrm{e}^{p \theta r_{0}}$, by Gronwall's inequality we get

$$
\left\|\bar{X}_{t}^{\xi}-\bar{X}_{t}^{\eta}\right\|_{\infty}^{p} \leq \mathrm{e}^{p \theta r_{0}-p\left\{\theta-\alpha_{1} \mathrm{e}^{p \theta r_{0}}\right\} t}\|\xi-\eta\|_{\infty}^{p} \leq e^{\left.p \theta r_{0}-p \kappa_{p}\right\} t}\|\xi-\eta\|_{\infty}^{p} .
$$

Combining this with (4.16) and using the semigroup property of $\bar{P}_{t}$, we find a constant $t_{1}>t_{0}$ such that

$$
\left.\left(\bar{P}_{t_{1}} f(\eta)\right)^{2} \leq\left(\bar{P}_{t_{1}} f^{2}(\xi)\right)\right) \mathrm{e}^{\varepsilon\|\xi-\eta\|_{\infty}^{2} / 2}, \quad \xi, \eta \in \mathscr{C}, f \in \mathscr{B}_{b}(\mathscr{C})
$$


This implies that the invariant probability measure $\bar{\mu}$ has full support on $\mathscr{C}$, so that there exists a constant $c>0$ such that

$$
\sup _{\bar{\mu}\left(|f|^{2}\right) \leq 1}\left(\bar{P}_{t_{1}} f(\xi)\right)^{2} \leq \frac{1}{\int_{\mathscr{C}} \mathrm{e}^{-\varepsilon\|\xi-\eta\|_{\infty}^{2} / 2} \bar{\mu}(\mathrm{d} \eta)} \leq c \mathrm{e}^{\varepsilon\|\xi\|_{\infty}^{2}}, \quad \xi \in \mathscr{C} .
$$

Therefore, $\bar{P}_{t_{1}}$ has a density $p_{t_{1}}(\xi, \eta)$ with respect to $\bar{\mu}$ satisfying

$$
\int_{\mathscr{C}} p_{t_{1}}(\xi, \eta)^{2} \bar{\mu}(\mathrm{d} \eta) \leq c \mathrm{e}^{\varepsilon\|\xi\|_{\infty}^{2}}, \quad \xi \in \mathscr{C} .
$$

Consequently, for any $\nu \in \mathscr{I}_{\varepsilon, R}, \bar{\nu}_{t_{1}}:=\mathscr{L}_{\bar{X}_{t_{1}}^{\nu}}$ has density

$$
h(\eta):=\int_{\mathscr{C}} p_{t_{1}}(\xi, \eta) \nu(\mathrm{d} \xi)
$$

with respect to $\bar{\mu}$ which satisfies

$$
\bar{\mu}\left(|h|^{2}\right) \leq \int_{\mathscr{C} \times \mathscr{C}} p_{t_{1}}(\xi, \eta)^{2} \nu(\mathrm{d} \xi) \bar{\mu}(\mathrm{d} \eta) \leq c \nu\left(\mathrm{e}^{\varepsilon\|\cdot\|_{\infty}^{2}}\right) \leq c R
$$

Combining this with (4.17) and noting that the Markov property of $\bar{X}_{t}$ implies that the law of $\bar{L}_{t}^{\bar{\nu}_{t_{1}}}$ coincides with that of

$$
\tilde{L}_{t}^{\nu}:=\frac{1}{t} \int_{t_{1}}^{t+t_{1}} \delta_{\bar{X}_{s}^{\nu}} \mathrm{d} s
$$

we prove

$$
\left\{\tilde{L}_{t}^{\nu}\right\}_{\nu \in \mathscr{I}_{\varepsilon, R}} \in \operatorname{LDP}(J) .
$$

On the other hand, for the distance $\rho$ in (4.3) we have

$$
\rho\left(\tilde{L}_{t}^{\nu}, \bar{L}_{t}^{\nu}\right) \leq \frac{2 t_{1}}{t}, \quad t>0 .
$$

So, by Lemma 3.5 and (4.18) we prove (I).

For (II). By $\left(H_{3}\right)$ and (2.13), there exist constants $c>0$ such that for $\theta \in\left[0, \lambda_{1}\right]$,

$$
\begin{aligned}
\left\|X_{t}^{\nu}-\bar{X}_{t}^{\nu}\right\|_{\infty} \mathrm{e}^{\theta t} & \leq \mathrm{e}^{\theta r_{0}} \sup _{s \in\left[\left(t-r_{0}\right)^{+}, t\right]}\left|X^{\nu}(s)-\bar{X}^{\nu}(s)\right| \mathrm{e}^{\theta s} \\
& \leq \mathrm{e}^{\theta r_{0}} \int_{0}^{t} \mathrm{e}^{\theta s}\left\{\alpha_{1}\left\|X_{s}^{\nu}-\bar{X}_{s}^{\nu}\right\|_{\infty}+\alpha_{2} \mathbb{W}_{p}\left(P_{s}^{*} \nu, \mu\right)\right\} \mathrm{d} s \\
& \leq c+\alpha_{1} \mathrm{e}^{\theta r_{0}} \int_{0}^{t} \mathrm{e}^{\theta s}\left\|X_{s}^{\nu}-\bar{X}_{s}^{\nu}\right\|_{\infty} \mathrm{d} s, \quad t \geq 0, \nu \in \mathscr{I}_{\varepsilon, R} .
\end{aligned}
$$

By Gronwall's inequality we obtain

$$
\sup _{\nu \in \mathscr{I}_{\varepsilon, R}}\left\|X_{t}^{\nu}-\bar{X}_{t}^{\nu}\right\|_{\infty} \leq c \exp \left[\left\{\alpha_{1} \mathrm{e}^{\theta r_{0}}-\theta\right\} t\right] \leq c \mathrm{e}^{-\kappa_{p} t}, \quad t>0 .
$$

This proves assertion (II). 


\subsection{Proof of Theorem 2.4}

By (2.16) , we take $\theta \in\left(0, \lambda_{1}\right]$ such that

$$
\theta \mathrm{e}^{-\theta r_{0}}-K_{2}-\alpha^{\prime}\|B\|>K_{3}
$$

For any $\alpha>0$, let

$$
\rho_{\alpha}\left(\xi_{1}, \xi_{2}\right):=\alpha\left\|\xi_{1}^{(1)}-\xi_{2}^{(1)}\right\|_{\infty}+\left\|\xi_{1}^{(2)}-\xi_{2}^{(2)}\right\|_{\infty}, \quad \xi_{1}, \xi_{2} \in \mathscr{C}
$$

We take $X_{0}, Y_{0} \in L^{2}\left(\Omega \rightarrow \mathscr{C}, \mathscr{F}_{0}, \mathbb{P}\right)$ such that $\mathscr{L}_{X_{0}}=\nu_{1}, \mathscr{L}_{Y_{0}}=\nu_{2}$ and

$$
\mathbb{W}_{p, \alpha}\left(\nu_{1}, \nu_{2}\right)^{p}=\mathbb{E} \rho_{\alpha}\left(X_{0}, Y_{0}\right)^{p}
$$

Let $X(t)$ and $Y(t)$ solves (2.14) with initial values $X_{0}$ and $Y_{0}$ respectively. Then $\left(H_{4}^{1}\right)$ implies $A_{1}-\delta \leq-\lambda_{1} \leq \theta$, so that

$$
\begin{aligned}
& \left|X^{(1)}(t)-Y^{(1)}(t)\right| \leq\left|\mathrm{e}^{\left(A_{1}-\delta\right) t}\left\{X^{(1)}(0)-Y^{(1)}(0)\right\}\right| \\
& \quad+\int_{0}^{t}\left|\mathrm{e}^{\left(A_{1}-\delta\right)(t-s)}\left\{\delta\left(X^{(1)}(s)-Y^{(1)}(s)\right)+B\left(X^{(2)}(s)-Y^{(2)}(s)\right)\right\}\right| \mathrm{d} s \\
& \leq \mathrm{e}^{-\theta t}\left|X^{(1)}(0)-Y^{(1)}(0)\right|+\int_{0}^{t} \mathrm{e}^{-\theta(t-s)}\left\{\delta\left|X^{(1)}(s)-Y^{(1)}(s)\right|+\|B\| \cdot\left|X^{(2)}(s)-Y^{(2)}(s)\right|\right\} \mathrm{d} s .
\end{aligned}
$$

Equivalently,

$$
\begin{aligned}
& \mathrm{e}^{\theta t}\left|X^{(1)}(t)-Y^{(1)}(t)\right| \leq\left|X^{(1)}(0)-Y^{(1)}(0)\right| \\
& \quad+\int_{0}^{t} \mathrm{e}^{\theta s}\left\{\delta\left|X^{(1)}(s)-Y^{(1)}(s)\right|+\|B\| \cdot\left|X^{(2)}(s)-Y^{(2)}(s)\right|\right\} \mathrm{d} s .
\end{aligned}
$$

Similarly, it follows from $A_{2} \leq-\lambda_{1} \leq-\theta$ and $\left(H_{4}^{2}\right)$ that

$$
\begin{aligned}
& \mathrm{e}^{\theta t}\left|X^{(2)}(t)-Y^{(2)}(t)\right| \leq\left|X^{(2)}(0)-Y^{(2)}(0)\right| \\
& +\int_{0}^{t} \mathrm{e}^{\theta s}\left\{K_{1}\left\|X_{s}^{(1)}-Y_{s}^{(1)}\right\|_{\infty}+K_{2}\left\|X_{s}^{(2)}-Y_{s}^{(2)}\right\|_{\infty}+K_{3} \mathbb{W}_{p, \alpha}\left(P_{s}^{*} \nu_{1}, P_{s}^{*} \nu_{2}\right)\right\} \mathrm{d} s .
\end{aligned}
$$

Combining these with $\alpha^{\prime} \geq \alpha$ and that $\lambda^{\prime}:=\frac{1}{2}\left\{\delta+K_{2}+\sqrt{\left(K_{2}-\delta\right)^{2}+4\|B\|}\right.$ satisfies

$$
\alpha^{\prime} \delta+K_{1}=\lambda^{\prime} \alpha^{\prime}, \quad \alpha^{\prime}\|B\|+K_{2}=\lambda^{\prime}>0
$$

we derive

$$
\begin{gathered}
\mathrm{e}^{\theta t} \rho_{\alpha^{\prime}}\left(X_{t}, Y_{t}\right) \leq \mathrm{e}^{\theta r_{0}} \sup _{s \in\left[t-r_{0}, t\right]}\left\{\alpha^{\prime}\left|X^{(1)}(s)-Y^{(1)}(s)\right|+\left|X^{(2)}(s)-Y^{(2)}(s)\right|\right\} \mathrm{e}^{\theta s} \\
\leq \mathrm{e}^{\theta r_{0}} \rho_{\alpha^{\prime}}\left(X_{0}, Y_{0}\right)+\mathrm{e}^{\theta r_{0}} \int_{0}^{t}\left\{\left(\delta \alpha^{\prime}+K_{1}\right)\left\|X_{s}^{(1)}-Y_{s}^{(1)}\right\|_{\infty}\right. \\
\left.+\left(\alpha^{\prime}\|B\|+K_{2}\right)\left\|X_{s}^{(2)}-Y_{s}^{(2)}\right\|_{\infty}+K_{3} \mathbb{W}_{p, \alpha}\left(P_{s}^{*} \nu_{1}, P_{s}^{*} \nu_{2}\right)\right\} \mathrm{d} s
\end{gathered}
$$




$$
=\mathrm{e}^{\theta r_{0}} \rho_{\alpha^{\prime}}\left(X_{0}, Y_{0}\right)+\mathrm{e}^{\theta r_{0}} \int_{0}^{t} \mathrm{e}^{\theta s}\left\{\lambda^{\prime} \rho_{\alpha^{\prime}}\left(X_{s}, Y_{s}\right)+K_{3} \mathbb{E}\left[\rho_{\alpha}\left(X_{s}, Y_{s}\right)\right]\right\} \mathrm{d} s .
$$

By Gronwall's lemma, for $\kappa:=\theta-\lambda^{\prime} \mathrm{e}^{\theta r_{0}}>0$ we have

$$
\rho_{\alpha^{\prime}}\left(X_{t}, Y_{t}\right) \leq \mathrm{e}^{\theta r_{0}-\kappa t} \rho_{\alpha^{\prime}}\left(X_{0}, Y_{0}\right)+\mathrm{e}^{\theta r_{0}} K_{3} \int_{0}^{t} \mathrm{e}^{-\kappa(t-s)} \mathbb{E}\left[\rho_{\alpha}\left(X_{s}, Y_{s}\right)\right] \mathrm{d} s
$$

Therefore, for any $\varepsilon>0$ there exists a constant $C(\varepsilon)>0$ such that

$$
\rho_{\alpha^{\prime}}\left(X_{t}, Y_{t}\right)^{p} \leq C(\varepsilon) \rho_{\alpha^{\prime}}\left(X_{0}, Y_{0}\right)^{p} \mathrm{e}^{-\kappa p t}+\frac{K_{3}^{p} \mathrm{e}^{\theta r_{0} p}(1+\varepsilon)}{\kappa^{p-1}} \int_{0}^{t} \mathrm{e}^{-\kappa(t-s)} \mathbb{E}\left[\rho_{\alpha}\left(X_{s}, Y_{s}\right)^{p}\right] \mathrm{d} s .
$$

Combining this with $\rho_{\alpha^{\prime}} \geq \rho_{\alpha}$ and $\mathbb{E}\left[\rho_{\alpha^{\prime}}\left(X_{t}, Y_{t}\right)^{p}\right]<\infty$ due to Theorem [3.1, we deduce from this and Gronwall's lemma that

$$
\begin{aligned}
& \mathbb{W}_{p, \alpha}\left(P_{t}^{*} \nu_{1}, P_{t}^{*} \nu_{2}\right)^{p} \leq \mathbb{E}\left[\rho_{\alpha^{\prime}}\left(X_{t}, Y_{t}\right)^{p}\right] \\
& \leq \frac{\alpha^{p} C(\varepsilon)}{\left(\alpha^{\prime}\right)^{p}} \mathbb{W}_{p, \alpha}\left(\nu_{1}, \nu_{2}\right)^{p} \exp \left[-\left(\kappa-(1+\varepsilon) K_{3}^{p} \mathrm{e}^{\theta r_{0} p} \kappa^{1-p}\right) t\right] .
\end{aligned}
$$

It is easy to see that (4.19) implies $\kappa>K_{3}^{p} \mathrm{e}^{\theta r_{0} p} \kappa^{1-p}$, so that by taking small enough $\varepsilon>0$ we prove

$$
\mathbb{W}_{p}\left(P_{t}^{*} \nu_{1}, P_{t}^{*} \nu_{2}\right) \leq c_{1} \mathrm{e}^{-c_{2} t}, \quad t \geq 0, \nu_{1}, \nu_{2} \in \mathscr{P}_{p}(\mathscr{C})
$$

for some constants $c_{1}, c_{2}>0$. Consequently, $P_{t}^{*}$ has a unique invariant probability measure $\bar{\mu}$ such that (2.17) holds.

Similarly, by $\left(H_{4}\right)$ and (2.17), we find a constant $C>0$ such that for any $X_{0}^{\nu}=\bar{X}_{0}^{\nu} \in$ $L^{p}\left(\Omega \rightarrow \mathscr{C}, \mathscr{F}_{0}, \mathbb{P}\right)$,

$$
\int_{0}^{\infty}\left\|X_{t}^{\nu}-\bar{X}_{t}^{\nu}\right\|_{\infty}^{2} \mathrm{~d} t \leq C, \quad \nu \in \mathscr{I}_{R, q} .
$$

Moreover, it is easy to see that (2.16) implies the condition in [1, Theorem 1.3] for the reference equation with $\bar{\mu}$ replacing the distribution of solution, so that $\bar{P}_{t}$ is hypecontractive (hence uniformly integrable in $L^{p}(\bar{\mu})$ for any $p>1$ ) for large $t>0$, and the Harnack in [1, Lemma 4.1] implies (4.16). Then the desired LDP can be proved in the same way as in the proof of Theorem 2.3.

\section{References}

[1] J. Bao, F.-Y. Wang, C. Yuan, Hypercontractivity for functional stochastic partial differential equations, Comm. Electr. Probab. 20(2015), 1-15.

[2] V. Bogachev, N.V. Krylov, M. Röckner, On regularity of transition probabilities and invariant measures of singular diffusions under minimal conditions, Comm. Part. Diff. Equat. 26(2001), 2037-2080.

[3] Da Prato, G., Zabczyk, J., Stochastic Equations in Infinite Dimensions, Cambridge University Press, Cambridge, 1992. 
[4] A. Dembo and O. Zeitouni, Large Deviations Techniques and Applications, Second Edition, Springer, New York. 1998.

[5] M. D. Donsker and S. R. S. Varadhan, Asymptotic evaluation of certain Markov process expectations for large time, I-IV, Comm. Pure Appl. Math. 28(1975), 1-47, 279-301; 29(1976), 389-461; 36(1983), 183-212.

[6] M. I. Freidlin, A. D. Wentzell, Random Perturbation of Dynamical Systems, Translated by J. Szuc, Springer, 1984.

[7] A. Guillin, F.-Y. Wang, Degenerate Fokker-Planck equations: Bismut formula, gradient estimate and Harnack inequality, J. Diff. Equat. 253(2012), 20-40.

[8] M. Hairer, On Malliavins proof of Hörmander's theorem, Bull. Sci. Math. 135(2011), 650-666.

[9] X. Huang, M. Röckner, F.-Y. Wang, Nonlinear Fokker-Planck equations for probability measures on path space and path-distribution dependent SDEs, Discrete Contin. Dyn. Syst. A. 39(2019), 3017-3035.

[10] M. Kac, Foundations of kinetic theory, In: Proceedings of the Third Berkeley Symposium on Mathematical Statistics and Probability, 1954-1955, vol. III. University of California Press, Berkeley and Los Angeles, 1956, pp. 171-197.

[11] E. Lanconelli, S. Polidoro, On a class of hypoelliptic evolution operator, Rend. Sem. Mat. Univ. Pol. Torino 52(1994), 29-63.

[12] H. P. McKean, A class of Markov processes associated with nonlinear parabolic equations, In: Proceedings of the National Academy of Sciences of the United States of America 56.6(1966), p. 1907.

[13] G. D. Reis, W. Salkeld, J. Tugaut, Freidlin-Wentzell LDP in path space for McKeanVlasov equations and the functional iterated logarithm law, Ann. Appl. Probab. 29(2019), 1487-1540.

[14] M. Röckner, F.-Y. Wang, L. Wu, Large deviations for stochastic generalized porous media equations, Stoch. Proc. Appl. 116(2006), 1677-1689.

[15] A.-S. Sznitman, Topics in Propagation of Chaos, Springer, 1991.

[16] F.-Y. Wang, Harnack inequalities and Applications for Stochastic Partial Differential Equations, Springer, 2013, Berlin.

[17] F.-Y. Wang, Hypercontractivity and applications for stochastic Hamiltonian systems, J. Funct. Anal. 272(2017), 5360-5383.

[18] F.-Y. Wang, Distribution dependent SDEs for Landau type equations, Stoch. Proc. Appl. 128(2018), 595-621. 
[19] F.-Y. Wang, X. Zhang, Derivative formula and applications for degenerate diffusion semigroups, J. Math. Pures Appl. 99(2013), 726-740.

[20] L. Wu, Some notes on large deviations of Markov processes, Acta Math. Sin. (English Ser.) 16(2000), 369-394.

[21] L. Wu, Uniformly integrable operators and large deviations for Markov processes, J. Funct. Anal. 172(2000), 301-376. 